\title{
ON THE SELBERG TRACE FORMULA IN THE CASE OF COMPACT QUOTIENT
}

\author{
BY NOLAN R. WALLACH
}

1. Introduction. Let $G$ be a connected unimodular Lie group. Let $\Gamma$ be a discrete subgroup of $G$ so that $\Gamma \backslash G$ is compact. We fix a Haar measure, $d g$, on $G$. Then $d g$ induces a $G$-invariant measure on $\Gamma \backslash G$. We can then form a unitary representation $\left(\pi_{\Gamma}, L^{2}(\Gamma \backslash G)\right)$ where $\left(\pi_{\Gamma}(g) f\right)(x)=f(x g)$ for $f \in L^{2}(\Gamma \backslash G), x \in \Gamma \backslash G, g \in G$. If $\phi \in C_{c}^{\infty}(G)$ (the space of all $C^{\infty}$ compactly supported complex valued functions on $G$ ) we can form

$$
\left(\pi_{\Gamma}(\phi) f\right)(x)=\int_{G} \phi(g) f(x g) d g .
$$

It is a standard fact (see $\$ 2)$ that $\pi_{\Gamma}(\phi)$ is of trace class. In particular, $\pi_{\Gamma}(\phi)$ is completely continuous for $\phi \in C_{c}^{\infty}(G)$. This implies that $L^{2}(\Gamma \backslash G)$ decomposes into an orthogonal direct sum of irreducible invariant subspaces, $\left\{H_{j}\right\}_{j=1}^{\infty}$ and for each $i$ there are only a finite number of $k$ so that $H_{\mathrm{j}}$ is equivalent with $H_{k}$ as a representation of $G$ (cf. Gelfand, Graev, PyateckiiShapiro [9]). Let $\hat{G}$ denote the set of equivalence classes of irreducible representations of $G$. Then we have observed that

$$
\pi_{\Gamma}=\sum_{\omega \in \hat{G}} N_{\Gamma}(\omega) \omega
$$

where $N_{\Gamma}(\omega)$ is a nonnegative integer. If $\omega \in \hat{G}$ we say that $\omega$ is of trace class if for each $(\pi, H) \in \omega, \phi \in C_{c}^{\infty}(G), \pi(\phi)=\int_{G} \phi(g) \pi(g) d g$ is a trace class operator on $H$. If $\omega \in \hat{G}$ is of trace class, then set $\Theta_{\omega}(\phi)=\operatorname{tr} \pi(\phi)$ for $(\pi, H) \in \omega$. The above observations imply that if $\omega \in \hat{G}$ and $N_{\Gamma}(\omega) \neq 0$, then $\omega$ is of trace class. We therefore see that if $\phi \in C_{c}^{\infty}(G)$, then

$$
\operatorname{tr} \pi_{\Gamma}(\phi)=\sum_{\omega \in \hat{G}} N_{\Gamma}(\omega) \Theta_{\omega}(\phi) .
$$

The numbers $N_{\Gamma}(\omega)$ have been the subject of a great deal of investigation in the last few years. In this article we will give a short survey of various techniques that have been used to study these integers. We will concentrate our attention on semisimple Lie groups, G. We will also, for most of the article, look at the easiest groups $\Gamma$. These groups have no elements of finite order other than the identity. Without this assumption many (interesting)

AMS(MOS) subject classifications (1970). Primary 10D20, 22E40, 43A15.

This address was given at the annual meeting of the American Mathematical Society in Washington, D.C. on January 24, 1975; received by the editors May 8, 1975. 
technicalities occur. We apologize to the reader for our avoidance of these technicalities.

It should also be pointed out that much of the important research on $L^{2}(\Gamma \backslash G)$ has been on the case where $\Gamma$ is discrete and $\Gamma \backslash G$ has finite volume relative to the measure on $\Gamma \backslash G$ induced by $d g$ (see Harish-Chandra [12], Duflo-Lebesse [4], Arthur [7]). The most noteworthy example is $G=S L(2, \mathbf{R}), \Gamma=S L(2, \mathbf{Z})(\mathbf{R}$ the reals, $\mathbf{Z}$ the integers $)$.

A serious reader of this article will be irritated with a noteworthy omission in this article. We will never give an example of a discrete group $\Gamma$ so that $\Gamma \backslash G$ is compact. The best we can say is that there are many of them. (See Mostow [29], Raghunathan [30].)

The author was introduced to the subject matter of this article by Professor Paul Sally. Many of the ideas in $\$ 9$ are an outgrowth of joint research with Sally. We also thank Professor Rioshi Hotta for having taught the author Matsushima's work on the Betti numbers of locally symmetric spaces. Finally, we thank Professor Kenneth Johnson for patiently teaching us his work on the Paley-Wiener problem for semisimple Lie groups.

2. The trace formula. In the Introduction we asserted that if $\phi \in C_{c}^{\infty}(G)$, then $\pi_{\Gamma}(\phi)$ is of trace class. We also computed a formula for $\operatorname{tr} \pi_{\Gamma}(\phi)$ in terms of the $N_{\Gamma}(\omega)$. We now compute another such formula first observed by Selberg. Let $f \in C^{\infty}(\Gamma \backslash G)$.

$$
\begin{aligned}
\pi_{\Gamma}(\phi) f(\Gamma x) & =\int_{G} f(\Gamma x g) \phi(g) d g=\int_{G} f(\Gamma g) \phi\left(x^{-1} g\right) d g \\
& =\int_{\Gamma \backslash G} \sum_{\gamma \in \Gamma} f(\Gamma \gamma g) \phi\left(x^{-1} \gamma g\right) d \dot{g} \\
& =\int_{\Gamma \backslash G} f(\Gamma g)\left(\sum_{\gamma \in \Gamma} \phi\left(x^{-1} \gamma g\right)\right) d \dot{g} .
\end{aligned}
$$

Thus if we set $K_{\phi, \Gamma}(x, g)=\sum_{\gamma \in \Gamma} \phi\left(x^{-1} \gamma g\right)$, then we note

$$
K_{\phi, \Gamma}(\tau x, g)=K_{\phi, \Gamma}(x, \tau g)=K_{\phi, \Gamma}(x, g)
$$

for $\tau \in \Gamma$. Thus $K_{\phi, \Gamma}: \Gamma \backslash G \times \Gamma \backslash G \rightarrow C$ is a $C^{\infty}$ function. We have

$$
\left(\pi_{\Gamma}(\phi) f\right)(x)=\int_{\Gamma \backslash G} K_{\Gamma, \phi}(x, y) f(y) d y .
$$

Standard theory now implies the trace class assertion and

$$
\operatorname{tr} \pi_{\Gamma}(\phi)=\int_{\Gamma \backslash G} K_{\Gamma, \phi}(\dot{x}, x) d x=\int_{\Gamma \backslash G}\left(\sum_{\gamma \in \Gamma} \phi\left(x^{-1} \gamma x\right)\right) d \dot{x} .
$$

Arguing now as in Gelfand, Graev, Pyateckili-Shapiro [9, p. 30] we can put this in the following form:

Theorem 2.1 (The tRACE FORMula). If $\phi \in C_{c}^{\infty}(G)$, then

$$
\operatorname{tr} \pi_{\Gamma}(\phi)=\sum_{[\gamma] \in[\Gamma]} \operatorname{vol}\left(\Gamma_{\gamma} \backslash G_{\gamma}\right) \int_{G_{\gamma} \backslash G} \phi\left(g^{-1} \gamma g\right) d \dot{g} .
$$


Here $[\Gamma]$ is the set of $\Gamma$-equivalence classes in $\Gamma$ and $[\gamma]$ is the $\Gamma$-equivalence class of $\gamma . G_{\gamma}$ is the centralizer of a representative of $[\gamma]=\left\{\tau \gamma \tau^{-1} \mid \tau \in \Gamma\right\}$. $\Gamma_{\gamma}=\Gamma \cap G_{\gamma}$. Here $\operatorname{vol}\left(\Gamma_{\gamma} \backslash G_{\gamma}\right)$ is the total measure of $\Gamma_{\gamma} \backslash G_{\gamma}$ and the measures are normalized by

and

$$
\int_{G} \psi(g) d g=\int_{G_{\gamma} \backslash G} \int_{G_{\gamma}} \psi(x g) d x d \dot{g}
$$

$$
\int_{G_{\gamma}} \eta(g) d g=\int_{\Gamma \gamma \backslash G_{\gamma}} \sum_{\tau \in \Gamma_{\gamma}} \eta(\tau g) d \dot{g} .
$$

3. How to use the trace formula. In this section we will assume that $G$ is a semisimple Lie group with finite center. We take $K \subset G$ a maximal compact subgroup. Then $X=G / K$ is the most general symmetric space of noncompact type.

For simplicity we assume that $\Gamma$ has no elements of finite order. This assumption implies (cf. Mostow [30]) that:

(a) If $\gamma \in \Gamma$ then $\operatorname{Ad}(\gamma)$ is a semisimple automorphism of the Lie algebra of $G$.

(b) $\Gamma$ acts freely on $X$.

Let $\hat{G}_{d}$ be the set of all equivalence classes of irreducible unitary representations equivalent with a subrepresentation of $L^{2}(G)$. Harish-Chandra $[13]$ has shown that $\hat{G}_{d} \neq \varnothing$ if and only if there is a maximal torus, $T$, of $K$ which is maximal abelian in $G$. In [13], Harish-Chandra also proves

THEOREM 3.1. Let $\omega \in \hat{G}_{d},(\pi, H) \in \omega$. Let $v, w \in H$ be $K$-finite (that is $\pi(K) v$ and $\pi(K) w$ are contained in finite dimensional subspaces of $H)$ and set $\psi(g)=\langle\pi(g) v, w\rangle$. Then if $\gamma$ is an element of $G$ so that $\operatorname{Ad}(\gamma)$ is semisimple, $\int_{G_{\gamma} \backslash G} \psi\left(g^{-1} \gamma g\right) d \dot{g}$ converges and is zero unless $\gamma \in\left\{\mathrm{gTg}^{-1} \mid \mathrm{g} \in G\right\}$.

Let $\hat{G}_{d}^{\prime}$ be the set of $\omega \in \hat{G}_{d}$ such that there are $v, w \in H((\pi, H) \in \omega)$ so that $\psi(\mathrm{g})$ (as above) is absolutely integrable. In Borel [2] it is proved that $\pi_{\Gamma}(\psi)$ is trace class and the trace formula applies.

Now if $\psi$ corresponds as above to $\omega \in G_{d}^{\prime}$, then $\Theta_{\eta}(\psi)=0$ if $\eta \neq \omega$. Thus $\operatorname{tr} \pi_{\Gamma}(\psi)=N_{\Gamma}(\omega) \Theta_{\omega}(\psi)$. On the other hand,

$$
\operatorname{tr} \pi_{\Gamma}(\psi)=\sum_{\gamma \in[\Gamma]} \operatorname{vol}\left(\Gamma_{\gamma} \backslash G_{\gamma}\right) \int_{G_{\gamma} \backslash G} \psi\left(g^{-1} \gamma g\right) d \dot{g} .
$$

But $\gamma \in \Gamma$ is conjugate to an element of $K$ if and only if $\gamma=I$. Thus we have

$$
N_{\Gamma}(\omega) \Theta_{\omega}(\psi)=\operatorname{vol}(\Gamma \backslash G) \psi(I) .
$$

Now the Schur orthogonality relations (see Harish-Chandra [10]) imply $\psi(I)=d(\omega) \Theta_{\omega}(\psi)$. Here $d(\omega)$ is defined by

$$
\int_{G}|\langle\pi(g) v, w\rangle|^{2} d g=\langle v, v\rangle\langle w, w\rangle d(\omega)^{-1}
$$

for $v, w \in H,(\pi, H) \in \omega, d(\omega)$ is called the formal degree of $\omega$. Clearly, if we take $v=w$ and $\langle v, v\rangle=1$ we have 
TheOREM 3.2 (LANGLANDs [26]). Suppose that $\Gamma$ is as above. If $\omega . \in \hat{G}_{d}^{\prime}$, then $N_{\Gamma}(\omega)=d(\omega) \operatorname{vol}(\Gamma \backslash G)$. (Here, of course, we take the same normalization of Haar measure to define $d(\omega)$ and $d \dot{g}$ on $\Gamma \backslash G$. Then the product $d(\omega) \operatorname{vol}(\Gamma \backslash G)$ is independent of normalization.)

If $\omega \in \hat{G}_{d}$, but not in $\hat{G}_{d}^{\prime}$, then this argument breaks down. The formula of 3.2 is so nice that one might hope that it is true for $\hat{G}_{d}$. Unfortunately, it is not. If we take $G=\operatorname{PSL}(2, \mathbf{R})$ (the group of holomorphic automorphisms of the upper half plane) and $\Gamma \subset G$ as above, then $\Gamma \backslash G / K(K=S O(2))$ is the most general Riemann surface of genus $g \geqq 2$. The Gauss-Bonnet theorem implies that we can normalize $d g$ so that $\operatorname{vol}(\Gamma \backslash G)=-\chi(\Gamma \backslash G / K)=2 g-2$.

$\hat{G}_{d}$ is naturally parametrized as $\left\{\omega_{n} \mid n \in \mathbf{Z}, n \neq 0\right\}$ and relative to this parametrization $\hat{G}_{d}=\left\{\omega_{1}, \omega_{-1}\right\} \cup \hat{G}_{d}^{\prime}, d\left(\omega_{n}\right)=|n| / 2$.

We therefore see that 3.2 implies

$$
N_{\Gamma}\left(\omega_{n}\right)=|n|(g-1)=d\left(\omega_{n}\right) \operatorname{vol}(\Gamma \backslash G) \text { if }|n| \geqq 2 .
$$

On the other hand, Langlands has shown (we will see this also in $\S 4$ ) that

$$
N_{\Gamma}\left(\omega_{ \pm 1}\right)=g=d\left(\omega_{ \pm 1}\right) \operatorname{vol}(\Gamma \backslash G)+1
$$

This certainly shows that the formula of Theorem 3.2 breaks down if $\omega \notin \hat{G}_{d}^{\prime}$. How do we interpret the "defect", $\left|d(\omega) \operatorname{vol}(\Gamma \backslash G)-N_{\Gamma}(\omega)\right|=1$ in formula (ii)? We will see that the interpretation of 1 is $N_{\Gamma}(1)$, the multiplicity of the trivial representation.

The numbers $N_{\Gamma}(\omega), \omega \in \hat{G}_{d}$ have been studied, using cohomological methods, by W. Schmid [33] and Hotta and Parthasarathy [17]. In the latter paper it is shown that the formula of Theorem 3.2 is true for a large class of elements of $\hat{G}_{d}$ not in $\hat{G}_{d}^{\prime}$.

4. Relations with the topology of $\Gamma \backslash G / K$. In the last section we noted that if $G=\operatorname{PSL}(2, \mathbf{R})$ and $\Gamma$ satisfies the conditions of the last section, then

$$
N_{\Gamma}\left(\omega_{1}\right)=N_{\Gamma}\left(\omega_{-1}\right)=g
$$

where $g$ is the genus of $\Gamma \backslash G / K$.

Matsushima [27] has a generalization of this formula which we will now describe.

Let $(\tau, V)$ be the complexification of the isotropy representation of $K$ on $T(G \backslash K)_{0}(0$ the coset $I \cdot K)$. Let $J_{p}$ be the set of equivalence classes of irreducible representations of $K$ that appear as a subrepresentation of $\Lambda^{\mathrm{P}} V$. If $\omega \in \hat{G}$ and $\gamma \in \hat{K}$, let $\left[\left.\omega\right|_{K}: \gamma\right]$ denote the multiplicity of $\gamma$ as a subrepresentation of any representative of $\omega$. Finally, let $\Omega$ be the Casimir operator of $G$ ( $\Omega$ is defined as follows: let $x_{1}, \cdots, x_{n}$ be a basis of $(S)$, the Lie algebra of $G$, let $x^{1}, \cdots, x^{n}$ be defined by $\operatorname{tr}$ ad $x_{i}$ ad $x^{j}=\delta_{i j} ;$ then $\left.\Omega=\sum x_{i} x^{i}\right)$. Let $\hat{G}_{0}=\{\omega \in$ $\hat{G} \mid \pi(\Omega)=0$ if $(\pi, H) \in \omega\}$.

Theorem 4.1 (Matsushima [27]). Let $b_{\mathrm{p}}(\Gamma \backslash G / K)=\operatorname{dim} H^{p}(\Gamma \backslash G / K, C)$. Then

$$
b_{\mathrm{p}}(\Gamma \backslash G / K)=\sum_{\omega \in \mathrm{G}_{0}} N_{\Gamma}(\omega)\left(\sum_{\gamma \in J_{\mathrm{p}}}\left[\left.\omega\right|_{K}: \gamma\right]\left[\Lambda^{p} V: \gamma\right]\right)
$$


This formula suggests that one should find all $\omega \in \hat{G}_{0}$ so that $\left[\left.\omega\right|_{K}: \gamma\right] \neq 0$ for some $\gamma \in J_{\mathrm{p}}$. Once such $\omega$ are found, then the next task it suggests is to find $N_{\Gamma}(\omega)$. We note that this formula implies that if for any $\omega \in \hat{G}_{0}, \gamma \in J_{p}$, $\left[\left.\omega\right|_{K}: \gamma\right]=0$, then $b_{p}(\Gamma \backslash G / K)=0$. Let us show how one can use this observation to prove that Betti numbers vanish.

Suppose that $G / K$ has a $G$-invariant complex structure. Then $V$ (above) splits into $V^{+} \oplus V^{-}$(corresponding to the holomorphic and antiholomorphic tangent spaces). $\Gamma \backslash G / K$ is then a projective algebraic variety (cf. Morrow [29]). We define $J_{p, q} \subset \hat{K}$ to be the set of equivalence classes of irreducible representations of $K$ appearing in $\Lambda^{p} V^{+} \otimes \Lambda^{q} V^{-}$. Then one has the analogous formula:

$$
b_{p, q}(\Gamma \backslash G / K)=\sum_{\omega \in G_{0}} N_{\Gamma}(\omega)\left(\sum_{\gamma \in J_{p, q}}\left[\left.\omega\right|_{K}: \gamma\right]\left[\Lambda^{p} V^{+} \otimes \Lambda^{q} V^{-}: \gamma\right]\right) .
$$

Theorem 4.2 (Hotta and Wallach [18]). Suppose that $G$ is simple and that $G / K$ has a $G$-invariant complex structure. Let $l=\operatorname{rank}(G / K)=$ split $\operatorname{rank}(G)$ (we will define this term in the next section). If $0<p<l$, then $\left\{\omega \in \hat{G}_{0} \mid\left[\left.\omega\right|_{K}: \gamma\right] \neq 0\right.$ for $\left.\gamma \in J_{0, p}\right\}=\varnothing$.

Using the above observations we have

Corollary 4.3. $\quad b_{0, p}(\Gamma \backslash G / K)=0$ for $0<p<\operatorname{rank}(G / K)$. Since

$$
b_{1}(\Gamma \backslash G / K)=b_{0,1}(\Gamma \backslash G / K)+b_{1,0}(\Gamma \backslash G / K)=2 b_{0,1}(\Gamma \backslash G / K),
$$

we have

Corollary 4.4 (Matsushima [26]). If $G / K$ has a $G$-invariant complex structure, $G$ is simple, and $\operatorname{rank}(G / K)>1$, then $b_{1}(\Gamma \backslash G / K)=0$.

Actually this theorem has been generalized by Kazdan [21] as follows:

THEOREM 4.5. If $G$ is a simple Lie group with split rank larger than 1 and if $\Gamma \backslash G$ is a discrete subgroup of $G$ so that $\operatorname{vol}(\Gamma \backslash G)<\infty$, then $\Gamma /[\Gamma, \Gamma]$ is finite.

This is a generalization of Corollary 4.4 since $\operatorname{rank}(\Gamma /[\Gamma, \Gamma])=b_{1}(\Gamma \backslash G / K)$ if $\Gamma$ is as in $\S 3$.

The proof of Theorem 4.2 also has implications for the rank 1 case. If $G$ is simple and $G / K$ has a $G$-invariant complex structure, then $G$ is locally isomorphic with $S U(n, 1)$. $S U(n, 1)$ is the subgroup of $S L(n+1, C)$ leaving the Hermitian form $\sum_{i=1}^{n}\left|z_{i}\right|^{2}-\left|z_{n+1}\right|^{2}$ invariant.

$G / K$ is the unit ball in $C^{n}$ under the action $g \cdot z=(\langle z, c\rangle+d)^{-1}(A z+b)$ where

$$
g=\left[\begin{array}{ll}
A & b \\
c^{*} & d
\end{array}\right]
$$

with $A, n \times n ; b ; n \times 1 ; c, n \times 1 ; d, 1 \times 1$. Here $C^{n}$ is looked upon as column vectors and $c^{*}$ is the conjugate transpose of $c . S U(1,1)$ is the twofold covering group of $\operatorname{PSL}(2, \mathbf{R})$.

One proves in this case

Proposition 4.6. There are elements $\omega_{\mathrm{p}, 0}$ and $\omega_{0, p}$ in $\hat{G}_{0}$ for 
$p=0,1,2, \cdots, n$ such that $\left\{\omega \in \hat{G}_{0} \mid\left[\left.\omega\right|_{K}: \gamma\right] \neq 0\right.$ for some $\left.\gamma \in J_{\mathrm{p}, q}\right\}=\left\{\omega_{\mathrm{p}, q}\right\}$ for $p$ or $q=0$. Furthermore, $J_{0, p}$ and $J_{p, 0}$ are singletons and $\left[\left.\omega_{0, p}\right|_{K}: \gamma\right]=1$ for $\gamma \in J_{0, p}$.

In the specific case $n=1$ we find $\omega_{0,1}=\omega_{1}$ and $\omega_{1,0}=\omega_{-1}$. Thus (ii) implies $N_{\Gamma}\left(\omega_{ \pm 1}\right)=g$. This gives another proof of this formula of Langlands.

5. An example. In this section we study the analogue of $\omega_{0,1}$ or $\omega_{1,0}$ for the Lorentz groups. Let $G=S O(n, 1)$ be the group of all $g \in S L(n+1, \mathbf{R})$ that leave the form $\sum_{i=1}^{n} x_{i}^{2}-x_{n+1}^{2}$ invariant. Then if $K=G \cap S O(n+1), G / K$ can be identified with the unit ball in $\mathbf{R}^{n}, B_{\mathbf{R}}^{n}$. This can be done via the action $g \cdot x=(\langle x, c\rangle+d)^{-1}(A x+b)$ where

$$
g=\left[\begin{array}{cc}
A & b \\
{ }^{c} c & d
\end{array}\right]
$$

with $A, n \times n ; c, b, n \times 1 ; d, 1 \times 1$. As before, $\mathbf{R}^{n}$ will be looked at as $n \times 1$ column vectors. It is easy to see that $G$ also acts on $S^{n-1}$, the unit sphere. For $f \in C^{\infty}\left(S^{n-1}\right)$ let $\left(\pi_{1}(g) f\right)(x)=f\left(g^{-1} \cdot x\right)$. If $f \in C^{\infty}\left(S^{n-1}\right)$, then $f=\sum_{p=0}^{\infty} f_{p}$ with $f_{p}$ a spherical harmonic of degree $p$. Let $(, \quad)$ be the $L^{2}$ inner product on $C^{\infty}\left(S^{n-1}\right)$ relative to the normalized standard volume element on $S^{n-1}$. Define for $f, g \in C^{\infty}\left(S^{n-1}\right)$,

$$
\langle f, g\rangle=\sum_{p=1}^{\infty}\left(\begin{array}{c}
n+p-2 \\
n-1
\end{array}\right) \quad\left(f_{p}, g_{p}\right) .
$$

Then $\langle$, $\rangle$ defines a pre-Hilbert space structure on $C^{\infty}\left(S^{n-1}\right) / C \cdot 1,1$ the constant function with value 1 on $S^{n-1}$. It can be shown that $\left\langle\pi_{1}(x) f, g\right\rangle=\left\langle f, \pi_{1}(x)^{-1} g\right\rangle$ for $f, g \in C^{\infty}\left(S^{n-1}\right)$ and that if $H_{1}$ is the Hilbert space completion of $\left(C^{\infty}\left(S^{n-1}\right) / C \cdot 1,\langle\right.$,$\rangle , then \left(\pi_{1}, H_{1}\right)$ is a unitary representation of $G$. For details see Johnson and Wallach [20].

Proposition 5.1 (Hotta and Wallach [18]). $\quad b_{1}\left(\Gamma \backslash C_{0} / K_{0}\right)=N_{\Gamma}\left(\pi_{1}\right) . G_{0}$ is the identity component of $G$, and $K_{0}=G_{0} \cap K$.

A unitary representation $(\pi, H)$ of $G$, a semisimple Lie group, is called tempered if $\Theta_{\pi}$ defines a tempered distribution on $G$ (that is, it extends to a continuous linear functional on the Schwartz space of $G$; see G. Warner [38]).

LEMMA 5.2. $\pi_{1}$ is a tempered representation of $(\operatorname{SO}(n, 1))_{0}$ if and only if $n=2$ or 3 .

See Johnson and Wallach [20] for a proof of this result.

If $G$ is a semisimple linear group, then a discrete subgroup, $\Gamma$, of $G$ is said to be arithmetic (see Borel [3]) if there is an injective finite dimensional real representation $(\rho, V)$ of $G$ and a basis of $V$ so that $\rho^{-1}(G L(N, Q))$ is dense in $G$, and if $\Gamma_{1}=\rho^{-1}(G L(N, Z))(N=\operatorname{dim} V)$, then $\Gamma \cap \Gamma_{1}$ is of finite index in $\Gamma$ and $\Gamma_{1}$.

THEOREM 5.3 (VINBERG [34]). If $n=3,4$, or 5 there is $\Gamma \backslash G_{0}(G=S O(n, 1))$ so that $\Gamma \backslash G_{0}$ is compact, $\Gamma$ is arithmetic and $\Gamma /[\Gamma, \Gamma]$ is infinite.

Using Theorem 5.3, we see that Proposition 5.1 implies that $N_{p}\left(\pi_{1}\right) \neq 0$ 
for the $\Gamma$ of Theorem 5.3. Lemma 5.2 says that $\pi_{1}$ is not tempered for $n \geq 4$. This gives the first example of a specific nontempered representation, $\omega$, with $N_{p}(\omega) \neq 0$. (Recently, Millson has shown that such $\Gamma$ exist for all $n$.)

6. The Selberg trace formula for (rank 1). Let $G$ be a connected semisimple Lie group with finite center. Let $K$ be as above and let $A, N$ be such that $G=K A N$ is an Iwasawa decomposition of $G$ (see, e.g., Helgason [15] or Wallach [35]). Then $A$ is a maximal vector subgroup of $G$ and $N$ is a maximal unipotent subgroup of $G$.

Definition 6.1. $G$ is said to be split rank 1 if $\operatorname{dim} A=1$.

We assume that $G$ is split rank 1 . Let $M$ be the centralizer of $A$ in $K$. We set $P=$ MAN. Then $P$ is a parabolic subgroup of $G$. Since $G$ has split rank 1 , all parabolic subgroups not equal to $G$ are gotten in this manner. (For the general definition see Warner [38, Chapter 1].)

Let $\mathfrak{a}$ be the Lie algebra of $A$. Then $\mathfrak{a}=\mathbf{R} H$. Let $n$ be the Lie algebra of $N$. We can normalize $H$ so that ad $\left.H\right|_{\mathfrak{n}}$ has eigenvalues 1 and possibly 2 (cf. Wallach [36]). Let $A^{+}=\{\exp t H \mid t>0\}$.

Let $p$ be the dimension of the eigenspace with eigenvalue 1 for ad $\left.H\right|_{n}, q$ the dimension of the eigenspace with eigenvalue 2 for ad $\left.H\right|_{n}$. Then $p>0$ and $0 \leqq q<p$. Set $\rho=\frac{1}{2}(p+2 q)$. If $\nu \in \mathbf{R}$ and $\xi \in \hat{M}$, let $H^{\xi}$ denote the space of functions

$$
\begin{gathered}
f: K \rightarrow H_{\xi} \quad\left(\left(\xi, H_{\xi}\right) \in \xi\right) \\
f(k m)=\xi(m)^{-1} f(k) \text { and } \int_{K}\|f(k)\|^{2} d k=\|f\|^{2}<\infty .
\end{gathered}
$$

If $f \in H^{\xi} \quad$ let $\quad f_{\nu}(k \exp t H n)=e^{-(i \nu+\rho) t} f(k), \quad k \in K, \quad t \in \mathbf{R}, \quad n \in N$. Set $\left(\pi_{\xi, \nu}(g) f\right)(k)=f_{\nu}\left(g^{-1} k\right)$. Then $\left(\pi_{\xi, \nu}, H^{\xi}\right)$ is a unitary representation of $G$.

If $f \in C_{0}^{\infty}(G)$ we define

$$
F_{f}(m \exp t H)=e^{t \rho} \int_{K \times N} f\left(k m a n k^{-1}\right) d n d k
$$

(here $d n$ is normalized so that

$$
\left.d g=e^{2 t \rho} d k d t d n, \quad g=k \exp t H n\right) .
$$

THEOREM 6.2. Let $\Theta_{\pi_{\xi, \nu}}=\Theta_{\xi, \nu}$ be the character of $\pi_{\xi, \nu}$. Then

$$
\Theta_{\xi, \nu}(f)=\int_{M} \int_{-\infty}^{\infty} F_{f}(m \exp t H) \operatorname{tr} \xi(m) e^{i \nu t} d t d m .
$$

Applying the Fourier inversion theorem and the Peter-Weyl theorem we have

LEMMA 6.3.

$$
F_{f}(m \exp t H)=\frac{1}{2 \pi} \sum_{\xi \in \hat{M}} \int_{-\infty}^{\infty} \Theta_{\xi, \nu}(f) e^{-i \nu t} \overline{\operatorname{tr} \xi(m)} d \nu .
$$

Now define for $m \in M, t \in \mathbf{R}$,

$$
D(m \exp t H)=e^{-t \rho}\left|\operatorname{det}\left(\left.\left(\operatorname{Ad}\left(m a_{t}\right)^{-1}-I\right)\right|_{\mathfrak{n}}\right)\right| .
$$


Clearly $D(m \exp t H) \neq 0$ if $t \neq 0$. A basic relation between $F_{f}$ and the trace formula is

Lemma 6.4 (CF. Wallach $[35,7.7 .10])$. If $t \neq 0$, then

$$
F_{f}(m a)=D(m a) \int_{G / A} f\left(g m a g^{-1}\right) d \dot{g} .
$$

(Here $d \dot{g}$ on $G / A$ is defined by

$$
\left.\int \phi(g) d g=\int_{G / A} \int_{-\infty}^{\infty} \phi(g \exp t H) d t d \dot{g} .\right)
$$

LEMMA 6.5. If $\mathrm{g} \in \mathrm{G}$ and $\mathrm{Ad}(\mathrm{g})$ is semisimple, then $\mathrm{g}$ is either conjugate to an element of $\mathrm{K}$ or an element of $\mathrm{MA}^{+}$.

This follows from the fact that $G$ has at most two conjugacy classes of Cartan subgroups (see Warner [38] for details).

Suppose now that $\Gamma \subset G$ satisfies the conditions of $\S 3$. If $\gamma \in \Gamma, \gamma \neq I$, then there is $x \in G$ so that $x \gamma x^{-1}=m_{\gamma} \exp t_{\gamma}, t_{\gamma}>0, t_{\gamma} \in \mathbf{R}, m_{\gamma} \in M$.

LEMMA 6.6. $t_{\gamma}$ depends only on $\gamma$ (not on the choice of $x$ or Iwasawa decomposition). Also $m_{\gamma}$ is determined up to conjugacy in $M$.

Proof. Since $\gamma$ is conjugate to an element of $\mathrm{MA}^{+}$we see that the eigenvalues of ad $\gamma$ are of the form $\delta, e^{t_{\gamma}} \lambda, e^{-t_{\gamma}} \mu, e^{2 t_{\gamma}} \eta$ or $e^{-2 t_{\gamma}} \psi$ with $1=|\lambda|=|\mu|=|\eta|=|\psi|=|\delta|$. Thus $e^{t_{\gamma}}$ is uniquely described as $(\max \{|\lambda| \mid \lambda \text { an eigenvalue of Ad } \gamma\})^{1 / 2}$ if ad $H$ has eigenvalue 2 or $\max \{|\lambda| \mid \lambda$ an eigenvalue of $\operatorname{Ad}(\gamma)\}$ otherwise. The second assertion is equally easy and we leave it to the reader.

Lemma 6.6 says that if $\gamma \in \Gamma, \xi \in \hat{M}$ and $\gamma \neq I$, then $D(\gamma)=D\left(m_{\gamma} \exp t_{\gamma} H\right)$, $t_{\gamma}$ and $\operatorname{tr} \xi\left(m_{\gamma}\right)$ are well defined (independent of $x \in G$ so that $\left.x \gamma x^{-1} \in M A\right)$.

If $h \in M A^{+}$and $G_{h}=\left\{g \in G \mid g h g^{-1}=h\right\}$, then

$$
\int_{G / A} f\left(g h g^{-1}\right) d g=\int_{G_{h} \backslash G} f\left(g^{-1} h g\right) d g \operatorname{vol}\left(G_{h} / A\right)
$$

since $G_{h} / A$ is compact. Let $u(\gamma)=\operatorname{vol}\left(G_{m_{\gamma}} \exp t_{\gamma} H / A\right)$. Combining Theorem 6.2 , Lemmas $6.3,6.5,6.6$ and the preceding observations, we have

Theorem 6.7 (The Selberg trace formula). If $f \in C_{c}^{\infty}(G)$, then

$$
\begin{aligned}
\sum_{\omega \in \hat{G}} N_{\Gamma}(\omega) \Theta_{\omega}(f)=\operatorname{vol}(\Gamma \backslash G) f(I) & +\frac{1}{2 \pi} \sum_{[\gamma] \in[\Gamma]-[I]} \operatorname{vol}\left(\Gamma_{\gamma} \backslash G_{\gamma}\right) D(\gamma)^{-1} \cdot u(\gamma) \\
& \cdot \sum_{\xi \in \hat{M}} \overline{\operatorname{tr} \xi\left(m_{\gamma}\right)} \int_{-\infty}^{\infty} \Theta_{\xi, \nu}(f) \cdot e^{-i t_{\gamma} \nu} d \nu .
\end{aligned}
$$

Theorem 6.7 says that the following problem is quite important to the computation of the $N_{\Gamma}(\omega)$.

Problem 6.8 (The Paley-Wiener problem). Describe the functions $\nu \rightarrow \Theta_{\xi, \nu}(f), \xi \in \hat{M}, \nu \in \mathbf{R}$ for $f \in C_{c}^{\infty}(G)$.

This problem has been solved by K. Johnson [19] up to a fairly touchy technical problem. There is, however, one case where the answer is exactly what one wishes. (For another, see §8.) 
Theorem 6.9 (S. Helgason [16], R. Gangolli [7]). If $\psi \in C_{c}^{\infty}(\mathbf{R})$ define

$$
\tilde{\phi}(\nu)=\int_{-\infty}^{\infty} \phi(t) e^{i v t} d t .
$$

A necessary and sufficient condition that a function $\psi: \mathbf{R} \rightarrow \mathbf{R}$ be of the form $\Theta_{1, \nu}(f)=\psi(\nu)$ for $f \in I_{c}^{\infty}(G)=\left\{f \in C_{c}^{\infty}(G) \mid f\left(k_{1} g k_{2}\right)=f(g)\right.$ for all $\left.k_{1}, k_{2} \in K\right\}$ is that $\psi=\tilde{\phi}$ for $\phi \in C_{c}^{\infty}(\mathbf{R})$, and $\phi(-t)=\phi(t)$ for all $t \in \mathbf{R}$.

If $\psi \in C_{\mathrm{c}}^{\infty}(\mathbf{R})$ let $f_{\psi} \in I_{\mathrm{c}}^{\infty}(G)$ be such that $\Theta_{1, \nu}\left(f_{\psi}\right)=\tilde{\psi}(\nu)$. The Plancherel theorem for spherical functions says

Theorem 6.10 (HARISH-Chandra [11], [13]). $f_{\phi}(I)=\int_{-\infty}^{\infty} \bar{\phi}(\nu) \cdot \mu_{1}(\nu) d \nu$ with $\mu_{1} \in C^{\infty}(\mathbf{R})$.

In order to derive a generalization of Selberg's original result for $\operatorname{PSL}(2, \mathbf{R})$, we need one more theorem.

Theorem 6.11 (Kostant [24]). To each $0 \leqq d_{p, q} \leqq \nu<c_{p, q}<\infty$ there exists an irreducible unitary representation of $G, \pi_{i v}$, so that if $f \in I_{c}^{\infty}(G), f=f_{\phi}$ for $\phi \in C_{c}^{\infty}(\mathbf{R})$, $\phi$ even, then

$$
\Theta_{\pi_{i \nu}}(f)=\tilde{\phi}(i \nu) .
$$

If $\omega \in \hat{G}$ and $\Theta_{\omega}(f) \neq 0$ for some $f \in I_{c}^{\infty}(G)$, then $\omega=\pi_{1, \nu}$ for some $\nu \in \mathbf{R}, \omega=1$ the trivial representation, or $\omega=\pi_{i v}$ for some $0 \leqq d_{p, q} \leqq \nu<c_{p, q}$. (Notice that $d_{p, q}$ and $c_{\mathrm{p}, q}$ depend only on $p, q$.)

Combining all of the above results we have

Corollary 6.12. Let $\phi \in C_{c}^{\infty}(\mathbf{R})$ be an even function. Then

$$
\begin{aligned}
\int_{-\infty}^{\infty} \phi(t) d t & +\sum_{\nu \in \mathbf{R}} N_{\Gamma}\left(\pi_{1, \nu}\right) \tilde{\phi}(\nu)+\sum_{d_{p, q} \leq \nu<c_{p, q}} N_{\Gamma}\left(\pi_{i \nu}\right) \tilde{\phi}(i \nu) \\
& =\operatorname{vol}(\Gamma \backslash G) \int_{-\infty}^{\infty} \tilde{\phi}(\nu) \mu_{1}(\nu) d \nu+\sum_{[\gamma] \in[\Gamma]-[r]} \operatorname{vol}\left(\Gamma_{\gamma} \backslash G_{\gamma}\right) D(\gamma)^{-1} u(\gamma) \phi\left(t_{\gamma}\right) .
\end{aligned}
$$

Although we will not give any applications of this result in this article (see Gelfand, et al. [9] for a discussion of this formula in the case $G=\operatorname{PSL}(2, \mathbf{R})$ ), we show how to use the results leading to this formula to prove that if $G=P S L(2, \mathbf{R})$, then

$$
N_{\Gamma}\left(\omega_{1}\right)=d\left(\omega_{1}\right) \operatorname{vol}(\Gamma \backslash G)+1 .
$$

In $\S 9$ we will show how to use this technique for $S U(2,1)$ and certain elements of $G_{d}-G_{d}^{\prime}$. The following technique is due to R. P. Langlands. Paul Sally taught the author this technique.

In this case $K=S O(2) / \pm I, M=\{I\}$. For $\vartheta \in \mathbf{R}$ let $k(\vartheta)$ be the rotation of $\mathbf{R}^{2}$ through the angle $\vartheta$. If $n \in \mathbf{Z}$ let $\xi_{n}(\vartheta)=e^{\text {in } \vartheta}$. Then $\left.\omega_{1}\right|_{K}=\sum_{n \geqq 1} \xi_{2 n}$.

Choose $f \in C_{c}^{\infty}(G)$ so that $f\left(k\left(\vartheta_{1}\right) g k\left(\vartheta_{2}\right)\right)=e^{-i\left(\vartheta_{1}+\vartheta_{2}\right)} f(g)$ and $\Theta_{\omega_{1}}(f)=1$. This is clearly possible. Now

$$
F_{f}(\exp t H)=e^{t p} \int_{K \times N} f\left(k \exp t H n k^{-1}\right) d k d n \quad \text { for } m \in M, t \in \mathbf{R} .
$$


Thus $F_{f}(\exp t H)$ is an element of $C_{c}^{\infty}(\mathbf{R})$. Theorem 6.9 says that there is $h \in I_{c}^{\infty}(G)$ so that $F_{h}=F_{f}$. Thus $F_{f-h}=0$.

According to the classification of irreducible unitary representatives of $\operatorname{PSL}(2, \mathbf{R})$ (see P. Sally [32] or Gelfand, et. al. [91]), if $\omega \in \hat{G}$ and $\Theta_{\omega}(f-h) \neq 0$, then $\omega=\omega_{1}$ or 1 . Thus we find using the arguments above that

$$
\int_{G}(f-h)(x) d x+N_{\Gamma}\left(\omega_{1}\right) \Theta_{\omega_{1}}(f-h)=\operatorname{vol}(\Gamma \backslash G)(f-h)(I) .
$$

Now $\Theta_{\omega_{1}}(h)=0\left(\left[\left.\omega\right|_{K}: 1\right]=0\right), \Theta_{\omega_{1}}(f)=1$. Furthermore, it can be shown that

$$
\int_{-\infty}^{\infty} F_{f}(\exp t H) e^{i \rho t} d t=\Theta_{\omega_{1}}(f)+\Theta_{\omega_{-1}}(f)+\int_{G} f(g) d g
$$

for $f \in C_{c}^{\infty}(G)$. Hence,

$$
0=\Theta_{\omega_{1}}(f-h)+\int_{G}(f-h)(g) d g .
$$

We therefore see that $\int_{G}(f-h)(g) d g=-1$. Finally,

$$
(f-h)(I)=\sum_{\omega \in \hat{G}_{d}} d(\omega) \Theta_{\omega}(f-h)
$$

by the Plancherel theorem for $\operatorname{PSL}(2, \mathbf{R}) \quad\left(F_{f-h}=0\right)$. We have already observed that $\Theta_{\omega}(f-g)=0$ if $\omega \neq \omega_{1}, \omega \in \hat{G}_{d}$ and $\Theta_{\omega_{1}}(f-g)=1$. Hence, we have

$$
-1+N_{\Gamma}\left(\omega_{1}\right)=d\left(\omega_{1}\right) \operatorname{vol}(\Gamma \backslash G) .
$$

This is the asserted formula.

7. $\hat{G}$ for $G=S U(2,1)$. In this section we give a list of the elements of $\hat{G}$ for $G=S U(2,1)$. We first describe the nonunitary principal series for $S U(2,1)=G$. Let $G$ act on $S^{3}=\left\{z \in C^{2}|| z \mid=1\right\}$ as follows:

$$
\mathrm{g} \cdot z=(\langle z, c\rangle+d)^{-1}(A z+b), \quad \mathrm{g}=\left[\begin{array}{ll}
A & b \\
c^{*} & d
\end{array}\right]
$$

(See §4.) Set $a(\mathrm{~g}, z)=\bar{d}-\langle z, b\rangle$ for $z \in S^{3}, \mathrm{~g} \in G$. If $k_{1}, k_{2} \in C$ and $k_{1}-k_{2} \in \mathbf{Z}$ (the integers), define

$$
\left(\pi_{k_{1}, k_{2}}(g) f\right)(z)=a(g, z)^{k_{1}} \overline{a(g, z)^{k_{2}}} f\left(g^{-1} \cdot z\right),
$$

for $f \in C^{\infty}\left(S^{3}\right), g \in G$.

Then $\pi_{k_{1}, k_{2}}(g)$ extends to a bounded operator on $L^{2}\left(S^{3}\right)=\mathscr{H}$ and $\left(\pi_{k_{1}, k_{2}}, \mathscr{H}\right)$ defines a continuous representation of $G$ for all $\left(k_{1}, k_{2}\right) \in C$ such that $k_{1}-k_{2} \in \mathbf{Z}$.

LEMMA 7.1. $\pi_{k_{1}, k_{2}}$ is reducible if and only if $\left(k_{1}, k_{2}\right) \in \mathbf{Z}^{2}$ and $\left(k_{1}, k_{2}\right) \neq(-1,-1)$.

We also note

LEMMA 7.2. $\left(\pi_{k_{1}, k_{2}}, \mathscr{H}\right)$ is a unitary representation (relative to the $L^{2}$-norm on $\mathscr{H})$ if and only if $-k_{1}-k_{2}=2+i v, \nu \in \mathbf{R}$.

The representations of Lemma 7.2 are just a reparametrization of the $\pi_{\xi, \nu}$ of $\S 6$.

Before going on with the analysis of the $\pi_{k_{1}, k_{2}}$, we should explain the 
notation. Let

$$
B=\left[\begin{array}{ccc}
2^{-1 / 2} & 0 & 2^{-1 / 2} \\
0 & 1 & 0 \\
2^{-1 / 2} & 0 & -2^{-1 / 2}
\end{array}\right] .
$$

Then $B^{2}=I$. $B(M A) B$ is a real form of the group of diagonal matrices in $S L(3, C)$. Let $\mathfrak{h}$ be the space of all trace zero, diagonal, $3 \times 3$, complex matrices. Then if $\mathbb{S}_{C}=s l(3, C)=\{X \mid X, 3 \times 3, \operatorname{tr} X=0\}, \mathfrak{h}$ is a Cartan subalgebra of $\mathbb{B S}_{C}$. We say that $\Lambda \in \mathfrak{h}^{*}$ is $G$-integral if $h \rightarrow \Lambda(B h B)$ is the differential of a quasi-character, $\xi_{\Lambda}$, of MA. Let $H$ be the element

$$
H=\left[\begin{array}{lll}
0 & 0 & 1 \\
0 & 0 & 0 \\
1 & 0 & 0
\end{array}\right] .
$$

Then $\mathfrak{a}=\mathbf{R} H$. Let $\Delta$ be the root system of $\mathbb{\mho}_{C}$ relative to $\mathfrak{h}$. Let $\Delta^{+}=\{\alpha \in$ $\Delta \mid \alpha(B h B)>0\}$. Let $\alpha_{1}, \alpha_{2}$ be the simple roots in $\Delta^{+}$. Let $\Lambda_{1}, \Lambda_{2}$ be the basic highest weights for this order. That is

$$
2\left\langle\Lambda_{i}, \alpha_{j}\right\rangle /\left\langle\alpha_{j}, \alpha_{j}\right\rangle=\delta_{i j}, \quad 1 \leqq i, j \leqq 2 .
$$

Here $\langle$,$\rangle is the dual bilinear form on \mathfrak{h}^{*}$ corresponding to the Killing form on $\mathbb{S}_{\mathrm{C}}$. Then $\Lambda \in \mathfrak{h}^{*}$ is $G$-integral if and only if $\Lambda=k_{1} \Lambda_{1}+k_{2} \Lambda_{2}, k_{i} \in C$, $i=1,2, k_{1}-k_{2} \in \mathbf{Z}$. For $\Lambda, G$-integral, let $X^{\Lambda}$ be the space of all $f \in C^{\infty}(G)$ such that

(1) $f(g m a)=\xi_{\Lambda}(m a) f(g)$,

(2) $\left(R_{Z} f\right)(g)=0$ for $Z \in B_{\mathfrak{n}}+B$.

Here $\mathfrak{N}^{+}$is the Lie algebra of upper triangular matrices with zeros on the diagonal. If $X \in \mathbb{B}$,

$$
\left(R_{X} f\right)(g)=\left.\frac{d}{d t} f(g \exp t X)\right|_{t=0}
$$

if $X \in \mathscr{S S}_{c}$,

$$
R_{\mathrm{X}_{1}+i \mathrm{X}_{2}} f=R_{\mathrm{X}_{1}} f+i R_{\mathrm{X}_{2}} f, \quad X=X_{1}+i X_{2}, X_{1}, X_{2} \in \text { G } s .
$$

If $f \in X^{\Lambda}, g \in G$, define $\left(T_{\Lambda}(g) f\right)(X)=f\left(g^{-1} X\right)$. Let

$$
K_{1}=\left\{\left[\begin{array}{l|l}
u & 0 \\
\hline 0 & 1
\end{array}\right] \mid u \in S U(2)\right\} .
$$

If $u \in S U(2)$,

$$
u=\left[\begin{array}{cc}
z_{1} & -\bar{z}_{2} \\
z_{2} & \bar{z}_{1}
\end{array}\right]=u\left(z_{1}, z_{2}\right) \quad \text { for }\left(z_{1}, z_{2}\right) \in S^{3} .
$$

It is easily seen that if $f \in X^{\Lambda}$ then $\left.f\right|_{K_{1}}$ determines $f$. If $f \in X^{\Lambda}$ define $\tilde{f}(z)=f(u(z)), z \in S^{3}$. Then if $\Lambda=k_{1} \Lambda_{1}+k_{2} \Lambda_{2},\left(T_{\Lambda}(g) f\right) u(z)=\left(\pi_{k_{1}, k_{2}}(g) \tilde{f}\right)(z)$ for $g \in G, z \in S^{3}$. Let $\rho=\frac{1}{2} \sum_{\alpha \in \Delta^{+}} \alpha$. We note that $\rho(B H B)=2$. We will now denote $\pi_{k_{1}, k_{2}}$ by $\pi_{\Lambda}, \Lambda=k_{1} \Lambda_{1}+k_{2} \Lambda_{2}$. We say $\Lambda$ is integral if $\Lambda=k_{1} \Lambda_{1}+k_{2} \Lambda_{2}$, $k_{1}, k_{2} \in \mathbf{Z}$. We can rephrase Lemma 7.1 to say 
Lemma 7.1'. $\quad \pi_{\Lambda}$ is reducible if and only if $\Lambda$ is integral and $\Lambda \neq-\rho$.

Now let $H^{\mathrm{p}, q}$ be the space of all polynomials $f$ on $\mathbf{C}^{2}$ which are homogeneous of degree $p$ in $z_{1}, z_{2}, q$ in $\bar{z}_{1}, \bar{z}_{2}$ (that is, $f(\lambda z)=\lambda^{\mathrm{p}} \bar{\lambda}^{\mathrm{q}} f(z)$ ) and such that

$$
\Delta f=\left(\frac{\partial^{2}}{\partial z_{1} \partial \bar{z}_{1}}+\frac{\partial^{2}}{\partial z_{2} \partial \bar{z}_{2}}\right) f=0 \text {. }
$$

Set $\mathscr{H}^{\mathrm{p}, \mathrm{q}}=\left.H^{\mathrm{p}, \mathrm{q}}\right|_{\mathbf{s}^{3}}$. Then $\mathscr{H}=\sum_{\mathrm{p}, \mathrm{q} \geqq 0} \mathscr{H}^{\mathrm{p}, \mathrm{q}}$ a unitary direct sum. Furthermore, $\left(\left.\pi_{\Lambda}\right|_{\kappa}, \mathscr{H}^{\mathrm{p}, \mathrm{q}}\right)$ is irreducible.

For $p, q \geqq 0$ set

$$
a_{p, q}(\Lambda)=\prod_{j=1}^{p} \frac{\left\langle\Lambda+(j+1) \rho, \alpha_{2}\right\rangle}{\left\langle-\Lambda+(j-1) \rho, \alpha_{1}\right\rangle} \cdot \prod_{j=1}^{q} \frac{\left\langle\Lambda+(j+1) \rho, \alpha_{1}\right\rangle}{\left\langle-\Lambda+(j-1) \rho, \alpha_{2}\right\rangle} .
$$

Here, as usual, $\prod_{j=u}^{v} a_{j}=1$ if $u>v$. (Note that if $\pi_{\Lambda}$ is irreducible then $a_{p, q}(\Lambda)$ is defined.)

For $f, g \in C^{\infty}\left(S^{3}\right)$ let

$$
\begin{gathered}
f=\sum f_{p, q}, \quad g=\sum g_{p, q}, \quad f_{p, q}, g_{p, q} \in \mathscr{H}^{p, q}, \\
\langle f, g\rangle_{\Lambda}=\sum_{p, q} a_{p, q}(\Lambda)\left\langle f_{p, q}, g_{p, q}\right\rangle .
\end{gathered}
$$

Lemma 7.3. Suppose that $\pi_{\Lambda}$ is irreducible. Then there exists a pre-Hilbert space structure on $C^{\infty}\left(S^{3}\right)$ so that $\left(\pi_{\Lambda}, C^{\infty}\left(S^{3}\right)\right)$ completes to a unitary representation if and only if one of the following holds:

$$
\begin{gathered}
-\Lambda(H)=2+i \nu, \quad \nu \in \mathbf{R} \\
\Lambda\left(H_{\alpha_{1}}\right)=\Lambda\left(H_{\alpha_{2}}\right) \quad \text { and }\left|2\left\langle\Lambda+\rho, \alpha_{1}+\alpha_{2}\right\rangle /\left\langle\alpha_{1}+\alpha_{2}, \alpha_{1}+\alpha_{2}\right\rangle\right|<2 ; \\
\Lambda\left(H_{\alpha_{1}}\right)-\Lambda\left(H_{\alpha_{2}}\right) \text { is odd and }\left|2\left\langle\Lambda+\rho, \alpha_{1}+\alpha_{2}\right\rangle /\left\langle\alpha_{1}+\alpha_{2}, \alpha_{1}+\alpha_{2}\right\rangle\right|<1 .
\end{gathered}
$$

In the latter two cases the pre-Hilbert space structure is defined by $\langle,\rangle_{\Lambda}$.

We are now left with an analysis of the reducible $\pi_{\Lambda}$. In this case it is easier to use the $T_{\Lambda}$ realization. For $f \in C^{\infty}(G)$ let $R_{1} f=R_{Z_{1}} f, R_{2} f=R_{Z_{2}} f$ where

$$
Z_{1}=B\left[\begin{array}{lll}
0 & 0 & 0 \\
1 & 0 & 0 \\
0 & 0 & 0
\end{array}\right] B, \quad Z_{2}=B\left[\begin{array}{lll}
0 & 0 & 0 \\
0 & 0 & 0 \\
0 & 1 & 0
\end{array}\right] B
$$

Lemma 7.4. Set $2\left\langle\Lambda+\rho, \alpha_{i}\right\rangle /\left\langle\alpha_{i}, \alpha_{i}\right\rangle=m_{i}, i=1,2$. (Note that $m_{i}=k_{i}+1$, $i=1,2$ if $\Lambda=k_{1} \Lambda_{1}+k_{2} \Lambda_{2}$.) If $m_{i} \in \mathbf{Z}, m_{i}>0$, then $R_{i}^{m_{i}} X^{\Lambda} \subset X^{S_{i}(\Lambda+\rho)-\rho}$. $\left(S_{i} \mu=\mu-\left(2\left\langle\mu, \alpha_{i}\right\rangle /\left\langle\alpha_{i}, \alpha_{i}\right\rangle\right) \alpha_{i}\right)$ Furthermore,

$$
R_{i}^{m_{i} \circ} T_{\Lambda}(g)=T_{S_{i}(\Lambda+\rho)-\rho}(g) \circ R_{i}^{m_{i}} .
$$

Using the map $f \rightarrow \tilde{f}$ of $X^{\Lambda}$ onto $C^{\infty}\left(S^{3}\right)$, we see that if $m_{i}>0, m_{i} \in \mathbf{Z}$ we can define $R_{i}^{m_{i}} f$ for $f \in C^{\infty}\left(S^{3}\right)$ by $\left(R_{i}^{m_{i}} f\right)^{\sim}=R_{i}^{m_{i}} \tilde{f}$. Then

$$
R_{i}^{m_{i}} \circ \pi_{\Lambda}(g)=\pi_{S_{i}(\Lambda+\rho)-\rho}(g) \circ R_{i}^{m_{i}} .
$$


LEMMA 7.5. For $j \geqq 0, j \in \mathbf{Z}$, let

$V_{j}^{+}=\left\{f \in C^{\infty}\left(S^{3}\right) \mid f_{p, q}=0\right.$ if $\left.q>j\right\}$,

$V_{j}^{-}=\left\{f \in C^{\infty}\left(S^{3}\right) \mid f_{\mathrm{p}, q}=0\right.$ if $\left.p>j\right\}$,

$W_{j}^{+}=\left\{f \in C^{\infty}\left(S^{3}\right) \mid f_{p, q}=0\right.$ if $\left.q \leqq j\right\}$,

$W_{j}^{-}=\left\{f \in C^{\infty}\left(S^{3}\right) \mid f_{p, q}=0\right.$ if $\left.p \leqq j\right\}$.

Suppose that $\Lambda \in \mathfrak{h}^{*}, \Lambda=k_{1} \Lambda_{1}+k_{2} \Lambda_{2}$.

(1) If $k_{1} \geqq 0, k_{1} \in \mathbf{Z}$, then $\operatorname{ker} R_{1}^{k_{1}+1}=V_{k_{1}}^{-}, R_{1}^{k_{1}+1} C^{\infty}\left(S^{3}\right)=W_{k_{1}}^{+}$.

(2) If $k_{2} \geqq 0, k_{2} \in \mathbf{Z}$, then $\operatorname{ker} R_{2}^{k_{2}+1}=V_{k_{2}}^{+}, R_{2}^{k_{2}+1} C^{\infty}\left(S^{3}\right)=W_{k_{2}}^{-}$.

Let 3 be the center of the universal enveloping algebra of $\mathscr{G}_{C}$. If $\Lambda \in \mathfrak{h}^{*}, \Lambda$ $G$-integral, it is not hard to see that $\pi_{\Lambda}(z) f=\chi_{\Lambda}(z) f, z \in \Re, \chi_{\Lambda}: 3 \rightarrow C$ an algebra homomorphism. Let $W_{C}$ be the Weyl group of $\Delta$. Then $W_{C}$ is generated by $S_{1}, S_{2}$. The general theory of the universal enveloping algebra implies that $\chi_{\Lambda}=\chi_{S(\Lambda+\rho)-\rho}$ for $S \in W_{C}$. We are almost ready to list the remaining elements of $\hat{G}$. We first need to look at certain systems of positive roots. Let

$$
\Delta_{1}^{+}=S_{1} S_{2} \Delta^{+}, \quad \Delta_{2}^{+}=S_{2} S_{1} \Delta^{+}
$$

and let

$$
S_{1} S_{2} S_{1} \Delta^{+}=S_{2} S_{1} S_{2} \Delta^{+}=-\Delta^{+}=\Delta_{3}^{+} .
$$

Let $\mathscr{F}$ denote the $G$-integral elements of $\mathfrak{h}^{*}$.

I. The holOMORPHIC DISCRETE SERIES. For $\Lambda \in \mathscr{F}$ and $\langle\Lambda+\rho, \alpha\rangle>0$ for $\alpha \in \Delta_{1}^{+}$let $\left(D_{\Lambda}^{+}, V_{+}^{\Lambda}\right)$ be defined as follows: $\Lambda=k_{1} \Lambda_{1}+k_{2} \Lambda_{2}$ with $k_{1}<0, k_{2} \geqq 0$. Let $V_{+}^{\Lambda}$ be the Hilbert space completion of $V_{k_{2}}^{+}$relative to $\langle f, g\rangle_{\Lambda}=$ $\sum a_{p, q}(\Lambda)\left\langle f_{p, q}, g_{p, q}\right\rangle . D_{\Lambda}^{+}(g)=\left.\pi_{\Lambda}(g)\right|_{v_{+}^{\Lambda}}$.

II. The ANTIHOlOMORPHiC Discrete SERIES. For $\Lambda \in \mathscr{F}$ and $\langle\Lambda+\rho, \alpha\rangle>0$ for $\alpha \in \Delta_{2}^{+}$let $\left(D_{\Lambda}^{-}, V_{-}^{\Lambda}\right)$ be defined as follows: $\Lambda=k_{1} \Lambda_{1}+k_{2} \Lambda_{2}, k_{1} \geq 0, k_{2}<0 . V_{-}^{\Lambda}$ is the Hilbert space completion of $V_{k_{1}}^{-}$relative to $\langle f, g\rangle_{\Lambda}=\sum a_{p, q}(\Lambda)\left\langle f_{p, q} g_{p, q}\right\rangle$. $D_{\Lambda}^{-}(g)=\left.\pi_{\Lambda}(g)\right|_{V^{\Lambda} \text {. }}$

III. The NONHOLOMORPhic DisCrete SERIES. For $\Lambda \in \mathscr{F}$ and $\langle\Lambda+\rho, \alpha\rangle>0$ for $\alpha \in \Delta_{3}^{+}$let $\left(D_{\Lambda}, W^{\Lambda}\right)$ be defined as follows: set $W^{\Lambda}=W_{-k_{1}-2}^{+} \cap W_{-k_{2}-2}^{-}$. Define

$$
b_{p, q}(\Lambda)=\prod_{j=-k_{2}}^{p} \frac{\left\langle\Lambda+(j+1) \rho, \alpha_{2}\right\rangle}{\left\langle-\Lambda+(j-1) \rho, \alpha_{1}\right\rangle} \prod_{j=-k_{1}}^{q} \frac{\left\langle\Lambda+(j+1) \rho, \alpha_{1}\right\rangle}{\left\langle-\Lambda+(j-1) \rho, \alpha_{2}\right\rangle}
$$

$p \geqq-k_{2}-1, q \geqq-k_{1}-1$. Let $W^{\Lambda}$ be the Hilbert space completion of $\mathcal{W}^{\Lambda}$ relative to $(f, g)_{\Lambda}=\sum b_{p, q}(\Lambda)\left\langle f_{p, q}, g_{p, q}\right\rangle . D_{\Lambda}(g)=\left.\pi_{\Lambda}(g)\right|_{W^{\Lambda}}$.

This gives a complete parametrization of $\hat{G}_{d}$. It is useful to give an alternate parametrization. We first observe that if $\Lambda \in \mathscr{F}^{+}, \Lambda=k_{1} \Lambda_{1}+k_{2} \Lambda_{2}$, then ker $R_{1}^{k_{1}+1} \cap$ ker $R_{2}^{k_{2}+1}$ is an invariant finite dimensional subspace of $\mathscr{H}$. Set $V^{\Lambda}=\operatorname{ker} R_{1}^{k_{1}+1} \cap \operatorname{ker} R_{2}^{k_{2}+1}$. Then $\left(\pi_{\Lambda}, V^{\Lambda}\right)$ is the finite dimensional irreducible representation of $G$ with lowest weight $-\Lambda$.

LEMMA 7.6.If $\Lambda \in \mathscr{F}^{+}=\left\{\Lambda \in \mathscr{F} \mid\langle\Lambda, \alpha\rangle \geqq 0\right.$ for $\left.\alpha \in \Delta^{+}\right\}$, let $G_{d, \Lambda}=\left\{\omega \in \hat{G}_{d} \mid\right.$ if 
$\left(\pi_{1} H\right) \in \omega, \pi(z)=\chi_{\Lambda}(z) I$ for $\left.z \in 8\right\}$. Then

$$
\hat{G}_{d, \Lambda}=\left\{D_{S_{1} S_{2}(\Lambda+\rho)-\rho}^{+}, D_{S_{2} S_{1}(\Lambda+\rho)-\rho}^{-}, D_{S_{1} S_{2} S_{1}(\Lambda+\rho)-\rho}\right\} .
$$

We can also describe $\hat{D}_{d}^{\prime}$.

LEMmA 7.7. $\hat{G}_{d}^{\prime} \cap \hat{G}_{d, \Lambda}=\hat{G}_{d, \Lambda} \quad$ if $\Lambda=k_{1} \Lambda_{1}+k_{2} \Lambda_{2}, \quad k_{1}, \quad k_{2} \geqq 2$. If $k_{1} \geqq 2$, $0 \leqq k_{2}<2$, then $\hat{G}_{d}^{\prime} \cap \hat{G}_{d, \Lambda}=\left\{D_{S_{2} s_{1}(\Lambda-\rho)-\rho}^{-}\right\}$. If $0 \leqq k_{1}<2$ and $k_{2} \geqq 2$, then $\hat{G}_{d}^{\prime} \cap \hat{G}_{d, \Lambda}=\left\{D_{S_{1} S_{2}(\Lambda+\rho)-\rho}^{+}\right\}$. If $0 \leqq k_{1}, k_{2}<2$, then $\hat{G}_{d}^{\prime} \cap \hat{G}_{d, \Lambda}=\varnothing$.

The next class of elements of $\hat{G}$ we describe are the irreducible constituents of reducible (unitary) principal series representations. From Lemmas 7.1 and 7.2 we see that the reducible unitary principal series consists of the $\left(\pi_{\Lambda}, \mathscr{H}\right)$ with $\Lambda$ integral, $\Lambda \neq-\rho$ and $\Lambda=k_{1} \Lambda_{1}+k_{2} \Lambda_{2}, k_{1}+k_{2}=-2$. We note that $k_{1}$ and $k_{2}$ cannot both be negative since $\Lambda$ is integral, $\Lambda \neq-\rho=-\Lambda_{1}-\Lambda_{2}$ and $k_{1}+k_{2}=-2$. There are thus two cases:

(i) $\Lambda=k_{1} \Lambda_{1}+k_{2} \Lambda_{2}, \quad k_{1} \geqq 0, \quad k_{1}+k_{2}=-2$. In this case if $H_{\Lambda}^{-}$is the $L^{2}-$ completion of $V_{k_{1}}^{-}, H_{\Lambda}^{-}$is an invariant subspace, and if $H_{\Lambda}^{+}$is the Hilbert space completion of $W_{k_{1}}^{+}$, then $H_{\Lambda}^{+}$is also invariant. $\mathscr{H}=H_{\Lambda}^{-} \oplus H_{\Lambda}^{+}, \pi_{\Lambda}=$ $\pi_{\Lambda}^{+} \oplus \pi_{\Lambda}^{-}, \pi_{\Lambda}^{ \pm}(g)=\left.\pi_{\Lambda}(g)\right|_{H_{\Lambda}^{ \pm}}$.

(ii) $\Lambda=k_{1} \Lambda_{1}+k_{2} \Lambda_{2}, k_{2} \geqq 0, k_{1}+k_{2}=-2, k_{i} \in \mathbf{Z}$. This time take $H_{\Lambda}^{+}$to be the $L^{2}$-completion of $V_{k_{2}}^{+}$and $H_{\Lambda}^{-}$the $L^{2}$-completion of $W_{k_{2}}^{-}$. Then $\mathscr{H}=H_{\Lambda}^{+} \oplus H_{\Lambda}^{-}$ and $\pi_{\Lambda}=\pi_{\Lambda}^{+} \oplus \pi_{\Lambda}^{-}$.

At this point we have described all of the tempered representations of $G$.

LEMMA 7.8. The tempered representations of $G$ consist of

(1) $\hat{G}_{d}$;

(2) the irreducible principal series: $\left(\pi_{\Lambda}, \mathscr{H}\right)$ with

$$
\operatorname{Re} 2\left\langle\Lambda, \alpha_{1}+\alpha_{2}\right\rangle /\left\langle\alpha_{1}+\alpha_{2}, \alpha_{1}+\alpha_{2}\right\rangle=-2
$$

and $\Lambda=-\rho$ or $\Lambda$ not integral;

(3) the irreducible constituents of reducible principal series: $\Lambda$ integral, $\Lambda \neq-\rho, 2\left\langle\Lambda, \alpha_{1}+\alpha_{2}\right\rangle /\left\langle\alpha_{1}+\alpha_{2}, \alpha_{1}+\alpha_{2}\right\rangle=-2$ and $\pi_{\Lambda}^{+}, \pi_{\Lambda}^{-}$.

If $G$ were $\operatorname{SL}(2, \mathbf{R})$ then the analogous list of Lemmas 7.8 and 7.3 with the addition of the trivial representation would completely describe $\hat{G}$. For $S U(2,1)$ there are more such "trivial representations". They are analogous to the representations of $S O(n, 1)$ of $\$ 5$. We now describe these extra representations.

$\left(T^{+}\right)$For $k \in \mathbf{Z}, k \geqq-1$, we define on $V_{0}^{+}$the Hermitian form

$$
\langle f, g\rangle_{k}=\sum_{p=0}^{\infty}\left(\begin{array}{l}
p+1 \\
k+2
\end{array}\right)\left\langle f_{p, 0}, g_{p, 0}\right\rangle ;
$$

then since $\left(\begin{array}{c}p+1 \\ k+2\end{array}\right)=0$ if $p<k+1$, we see that $\langle, \quad\rangle_{k}$ induces a pre-Hilbert space structure on $V_{0}^{+} / V_{k}^{-} \cap V_{0}^{+}\left(V_{-1}^{-}=0\right)$. Let $Z_{k}^{+}$denote the Hilbert space completion of $V_{0}^{+} / V_{k}^{-} \cap V_{0}^{+}$. Let $T_{k}^{+}(g)$ be the operator induced by $\left.\pi_{k \Lambda_{1}}(g)\right|_{V_{0}^{+}}$. Then $\left(T_{k}^{+}, Z_{k}^{+}\right)$defines a unitary representation of $G$.

$(T)$ For $k \in \mathbf{Z}, k \geqq-1$, define on $V_{0}^{-}$the form

$$
(f, g)_{k}=\sum_{p=0}^{\infty}\left(\begin{array}{l}
p+1 \\
k+2
\end{array}\right)\left\langle f_{0, p}, g_{0, p}\right\rangle .
$$


Take $Z_{k}^{-}$to be the Hilbert space completion of $V_{0}^{-} / V_{0}^{-} \cap V_{k}^{+}\left(V_{-1}^{+}=0\right)$ relative to $(, \quad)_{k}$. Let $T_{k}^{-}$be defined as in $\left(T^{+}\right)$(with $\Lambda_{1}$ replaced by $\Lambda_{2}$ ).

Then $\left(T_{k}^{-}, Z_{k}^{-}\right)$is a unitary representation of $G$.

The representations listed combined with the trivial representation completely describe $\hat{G}$.

In $\S 9$ we will use this description of $\hat{G}$ in the trace formula. To do this we need a list of the $K$-types in each element of $\hat{G}$. We will also need to know where the various elements of $\hat{G}$ appear in the composition series of the $\pi_{\Lambda}$ (ignoring the unitary structures). To describe the $K$-types we first note that

$$
K=\left\{\left[\begin{array}{c|c}
u & 0 \\
\hline 0 & (\operatorname{det} u)^{-1}
\end{array}\right] \mid u \in U(2)\right\} .
$$

Thus $K=S^{1} \cdot K_{1}$. That is, if $u \in K$, then

$$
u=\left[\begin{array}{c|c}
e^{i \vartheta} u_{1} & 0 \\
\hline 0 & e^{-2 i \vartheta}
\end{array}\right], \quad u_{1} \in S U(2), \vartheta \in \mathbf{R} .
$$

The representations of $K_{1}=S U(2)$ are parametrized by their dimensions. Let $\tau_{p}$ denote the irreducible representation of $K_{1}$ of dimension $p+1$. Let $\tau_{p}^{l}$ be defined by the following:

$$
\begin{gathered}
\tau_{\mathrm{p} \mid K_{1}}^{l}=\tau_{\mathrm{p}}, \\
\tau_{p}^{l}\left(\left[\begin{array}{c|c}
e_{I}^{i \vartheta} & 0 \\
\hline 0 & e^{-2 i \vartheta}
\end{array}\right]\right)=e^{-i l \vartheta} I .
\end{gathered}
$$

Of course the only $\tau_{p}^{l}$ that are representations of $U(2)=K$ are the ones such that $l \equiv p \bmod 2$.

Lemma 7.9 Let $\Lambda \in \mathfrak{h}^{*}$ be $G$-integral. Set $l=k_{2}-k_{1}, \Lambda=k_{1} \Lambda_{1}+k_{2} \Lambda_{2}$ (then $l \in \mathbf{Z})$. Then

$$
\left(\left.\pi_{\Lambda}\right|_{K}, \mathscr{H}^{p, q}\right) \equiv \tau_{p+q}^{2 l+3(p-q)}
$$

( $\equiv$ indicates $\mathrm{K}$-equivalence).

This lemma allows one to determine completely the K-types in each $\omega \in \hat{G}$. If $\pi$ is a representation of $G$ (not necessarily unitary) then we say that $\pi \subset \pi_{\Lambda}$ if $\pi$ is infinitesimally equivalent with a subquotient of $\pi_{\Lambda}$. For our purposes this can be taken to mean that if $H$ is the representation space for $\pi$, then there is a dense subspace $H_{0} \subset H$ and $\mathscr{H} \supset V_{1} \supset V_{2}, V_{i}$ closed invariant spaces of $\mathscr{H}$ and $A: H_{0} \rightarrow V_{1} / V_{2}$ an injective intertwining operator of $\pi$ with the induced action of $\pi_{\Lambda}$ on $V_{1} / V_{2}$ so that $A\left(H_{0}\right)$ is dense in $V_{1} / V_{2}$.

Set $S_{0}=S_{1} S_{2} S_{1}=S_{2} S_{1} S_{2}$ : Then if $\pi_{\Lambda}$ is irreducible and if $\pi_{\Lambda} \subset \pi_{\mu}$ for some $\mu$, then $\mu=S_{0}(\Lambda+\rho)-\rho$. This can be proved in this case by comparing representations of $K_{1}$.

We may therefore confine our attention to $\hat{G}_{d}$, the $\pi_{\Lambda}^{+}, \pi_{\Lambda}^{-}$and the $T_{k}^{ \pm}$, $k \geqq-1$.

LEMMA 7.10. If $\Lambda=k_{1} \Lambda_{1}+k_{2} \Lambda_{2}, k_{i} \geqq 0, k_{i} \in \mathbf{Z}$, then

(1) $D_{S_{0}(\Lambda+\rho)-\rho} \subset \pi_{\mu}$ if and only if $\mu=S(\Lambda+\rho)-\rho$ for some $s \in W_{C}$. 
(2) $D_{S_{1} S_{2}(\Lambda+\rho)-\rho}^{+} \subset \pi_{\mu}$ if and only if $\mu=S_{1}(\Lambda+\rho)-\rho$, or $\mu=S_{1} S_{2}(\Lambda+\rho)-\rho$.

(3) $D_{S_{2} S_{1}(\Lambda+\rho)-\rho}^{-} \subset \pi_{\mu}$ if and only if $\mu=S_{2}(\Lambda+\rho)-\rho$ or $\mu=S_{2} S_{1}(\Lambda+\rho)-\rho$.

LemMA 7.11 Let $\Lambda=k_{1} \Lambda_{1}+k_{2} \Lambda_{2}, k_{1}, k_{2} \in \mathbf{Z}, k_{1}+k_{2}=-2$ and $\Lambda \neq-\rho$.

(1) Suppose $k_{1} \geqq 0$. Then $\pi_{\Lambda}^{-} \subset \pi_{\mu}$ if and only if $\mu=\Lambda . \pi_{\Lambda}^{+} \subset \pi_{\mu}$ if and only if $\mu=S(\Lambda+\rho)-\rho$ for some $S \in W_{C}$ (note that $S_{0}(\Lambda+\rho)-\rho=\Lambda$ ).

(2) Suppose $k_{2} \geqq 0$. Then $\pi_{\Lambda}^{+} \subset \pi_{\mu}$ if and only if $\mu=\Lambda$. $\pi_{\Lambda}^{-} \subset \pi_{\mu}$ if and only if $\mu=S(\Lambda+\rho)-\rho$ for some $S \in W_{C}\left(S_{0}(\Lambda+\rho)-\rho=\Lambda\right)$.

We are left with the representations $T_{k}^{+}$and $T_{k}^{-}$.

LEMMA 7.12. (1) If $k \geq 0$ then $T_{k}^{+} \subset \pi_{\Lambda}$ if and only if $\Lambda=S_{1}\left(k \Lambda_{1}+\rho\right)-\rho$, $S_{0}\left(k \Lambda_{1}+\rho\right)-\rho, S_{1} S_{2}\left(k \Lambda_{2}+\rho\right)-\rho$ or $k \Lambda_{1}$.

(2) If $k \geqq 0$ then $T_{k}^{-} \subset \pi_{\Lambda}$ if and only if $\Lambda=S_{2}\left(k \Lambda_{2}+\rho\right)-\rho, S_{0}\left(k \Lambda_{2}+\rho\right)-\rho$, $S_{2} S_{1}\left(k \Lambda_{2}+\rho\right)-\rho$ or $k \Lambda_{2}$.

(3) $T_{-1}^{+} \subset \pi_{\Lambda}$ if and only if $\Lambda=S\left(-\Lambda_{1}+\rho\right)-\rho$ for some $S \in W_{C}$.

(4) $T_{-1}^{-} \subset \pi_{\Lambda}$ if and only if $\Lambda=S\left(-\Lambda_{2}+\rho\right)-\rho$ for some $S \in W_{C}$.

8. The image of $F_{f}$. In order to apply the results of $\$ 7$ to the trace formula, we will need some more information on $F_{f}$. We assume that $G$ is a simple Lie group of split rank 1 and has finite center. We retain the notation of $\S 6$. The main result of this section is

THEOREM 8.1. Let $\tau$ be a one dimensional representation of $K$. If $\phi \in$ $C_{c}^{\infty}(M A)$ and $\phi(m a)=\tau(m) \phi(a), \phi(a)=\phi\left(a^{-1}\right)$ for $a \in A$, then there exists $f \in C_{c}^{\infty}(G)$ with $f\left(k_{1} g k_{2}\right)=\tau\left(k_{1}\right) f(g) \tau\left(k_{2}\right)$ so that $F_{f}=\phi$.

This result has been proved in the case $G=S U(2,1)$ by Gupta (Thesis, University of Washington). His proof is substantially the same as the one outlined below.

We sketch a proof of this result. The idea of the proof is a modification of Helgason's technique for proving Theorem 6.9. We first note that if $\tau$ is the trivial representation, then Theorem 8.1 is a special case of Theorem 6.9.

We therefore assume that $\tau$ is nontrivial (the technique we describe works for the trivial representation and is actually easier in that case). Since $\tau$ is nontrivial, $K$ cannot be semisimple. Since $G$ is simple and split rank 1 , this implies $G$ is locally isomorphic with $S U(n, 1)$. Let $\xi=\left.\tau\right|_{M}$. For $\nu \in \mathbf{R}$ let $\left(\pi_{\xi, \nu}, H^{\xi}\right)$ be as in the beginning of $\S 6.3$. We note that for $\nu \in C,\left(\pi_{\xi, \nu}, H^{\xi}\right)$ makes perfectly good sense; it is just not necessarily a unitary representation. Let $C_{c}^{\infty}(\tau: G: \tau)$ be the space of all $C_{c}^{\infty}$ functions $f$ such that $f\left(k_{1} g k_{2}\right)=\tau\left(k_{1}\right) f(g) \tau\left(k_{2}\right)$. Let $E_{\tau}: H^{\xi} \rightarrow H_{\tau}^{\xi}$ be the projection onto the (one dimensional) subrepresentation of $\left(\left.\pi_{\xi, \nu}\right|_{K}, H^{\xi}\right)$ equivalent to $\tau$.

Let $E_{\tau}(\nu: g)=E_{\tau} \pi_{\xi, \nu}(g) E_{\tau}$ for $v \in C$. Then the Plancherel formula for $G$ says that there is a positive function, $\mu_{\tau}$, on $\mathbf{R}$ so that (see Harish-Chandra [14], G. Warner [37, Epilogue])

$$
f(I)=\sum_{\omega \in \widehat{G}_{d}} \Theta_{\omega}\left(R_{g} f\right)+\int_{-\infty}^{\infty} \alpha(\nu) E_{\tau}(v: g) \mu_{\tau}(\nu) d \nu .
$$


Here $\alpha(\nu)$ is, up to a constant independent of $\nu, \tau$, given as

$$
\alpha(\nu)=\int_{-\infty}^{\infty} F_{f}\left(a_{t}\right) e^{i t \nu} d t
$$

Since $\tau$ is one dimensional it is easily checked that $\alpha(\nu)=\alpha(-\nu)$. Since $\left.F_{f}\right|_{A}$ has compact support it follows that $\alpha$ extends to an entire function on $C$ and there is $T>0$ so that if $\varepsilon>0, k>0$ are given, there is $C_{k, \varepsilon}$ such that

(i) $|\alpha(\nu)| \leqq C_{k, \varepsilon}(1+|\nu|)^{-k} \exp ((T+\varepsilon)|\operatorname{Im} \nu|)$,

(ii) $\alpha(\nu)=\alpha(-\nu)$.

Let $\mathscr{P}(T)$ be the space of all entire functions on $C$ satisfying (i), (ii). To prove Theorem 8.1 we need only show that if $\alpha \in \mathscr{P}(T)$ and if

$$
f_{\alpha}(g)=\int_{-\infty}^{\infty} \alpha(\nu) E_{\tau}(\nu: g) \mu_{\tau}(\nu) d \nu
$$

then there exist $\omega_{1}, \cdots, \omega_{k} \in \hat{G}_{d}$ and $\phi_{1}, \cdots, \phi_{k}$ matrix entries of $\omega_{1}, \cdots, \omega_{k}$ so that $f_{\alpha}-\sum \phi_{i} \in C_{c}^{\infty}(\tau: G: \tau)$. Here we use the fact that for such $\phi_{j}, F_{\phi_{j}}=0$ (Harish-Chandra [13]).

To do this we study in more detail the functions $E_{\tau}(\nu: g)$. In Wallach [35] it is shown that if $\Delta(t)=(\sin h t)^{2 n-2} \sin h 2 t$, and if $\Phi_{\tau}(\nu: t)=\Delta(t)^{1 / 2} E_{\tau}\left(\nu: a_{t}\right)$ for $t>0$, then there is $Q_{\tau} \in C^{\infty}((0, \infty))$ so that

$$
\begin{gathered}
Q_{\tau}(t)=\sum_{k=1}^{\infty} a_{k} e^{-k t} \quad \text { for } t \geqq 1, \\
-\frac{d^{2}}{d t^{2}} \Phi_{\tau}(v: t)+Q_{\tau}(t) \Phi_{\tau}(v: t)=\nu^{2} \Phi_{\tau}(v: t) .
\end{gathered}
$$

In Wallach [35, Appendix], the following result was proved (see also Dunford and Schwartz [5]).

LEMMA 8.2. There exists an $\varepsilon>0$ and a continuous function $\sigma: \bar{H}_{\varepsilon} \times(0, \infty) \rightarrow C\left(H_{\varepsilon}=\{z \in C \mid \operatorname{Im} z>-\varepsilon\}\right)$ so that

(1) $t \rightarrow \sigma(\nu: t)$ is $C^{\infty}$ for $\nu \in \bar{H}_{\epsilon}, t>0$.

(2) $|\sigma(\nu: t)| \leqq 2$ for $t \geqq a_{0}$ for $\nu \in \bar{H}_{0}, a_{0}$ independent of $\nu$.

$$
-\frac{d^{2}}{d t^{2}}\left(e^{i \nu t} \sigma(\nu: t)\right)+Q_{\tau}(t) e^{i \nu t} \sigma(\nu: t)=\nu^{2} e^{i \nu t} \sigma(\nu: t), \quad \nu \in \bar{H}_{\varepsilon} .
$$

(4) $\lim _{t \rightarrow \infty, t>0} \sigma(\nu: t)=1$ for $\nu \in \bar{H}_{\varepsilon}$.

(5) $\nu \rightarrow \sigma(\nu: t)$ is holomorphic for $\nu \in H_{\varepsilon}$.

This result was used in Wallach [35] to show that if $\nu \in \mathbf{R}, v \neq 0$

$$
\Phi_{\tau}\left(\nu: a_{t}\right)=e^{i \nu t} C_{\tau}(\nu) \sigma(\nu: t)+e^{-i \nu t} C_{\tau}(-\nu) \sigma(-\nu: t)
$$

for $t>0$. It was also shown that if $\operatorname{Im} \nu<0$, then

$$
C_{\tau}(\nu)=\int_{\bar{N}} \exp (-(\rho+i \nu)(H(\bar{n}))) \tau(K(\bar{n}))^{-1} d \bar{n} .
$$

(Here $\bar{N}=\vartheta(N), \vartheta(g)={ }^{t} \bar{g}^{-1}$ for $g \in S U(n, 1)$ for an appropriate choice of $N$. Also if $g \in G, g=K(g) \exp (H(g) H) n(g)$ with $K(g) \in K, n(g) \in N$.) Now $K$ is locally isomorphic to $U(n)$. Let $\tau_{k}$ denote the $k$ th power of the determinant 
function (for coverings of $S U(n, 1), k$ can be a rational number). Then $\tau_{k}$ describes the typical one dimensional representation of $K$.

LEMma 8.3. There is a constant, $c(n)=C$, depending only on $n$ so that

$$
C_{\tau_{k}}(\nu)=C \frac{\Gamma(i \nu / 2) \Gamma\left(i \nu / 2+\frac{1}{2}\right)}{\Gamma((n+i \nu+k) / 2) \Gamma((n+i \nu-k) / 2)}
$$

where $\Gamma$ is the classical gamma function (cf. Whittaker and Watson [38]).

LEMMA 8.4 (HARISH-ChandRA [14], G. WARner [37]). $\mu_{\tau}(\nu)=$ $\left(C_{\tau}(-\nu) C_{\tau}(\nu)\right)^{-1}$ for $\nu \in \mathbf{R}$.

Lemma 8.4 is a very special case of the Maas-Selberg relations of Harish-Chandra. A proof of this result can also be found in Knapp-Stein [22].

The above results say that

$$
\Delta(t)^{1 / 2} E_{\tau}\left(\nu: a_{t}\right) \mu_{\tau}(\nu)=e^{i \nu t} C_{\tau}(-\nu)^{-1} \sigma(\nu: t)+e^{-i \nu t} C_{\tau}(\nu)^{-1} \sigma(-\nu: t)
$$

for $\nu \in \mathbf{R}$. Thus,

$$
\begin{aligned}
\Delta(t)^{1 / 2} f_{\alpha}\left(a_{t}\right)= & \int_{-\infty}^{\infty} e^{i \nu t} C_{\tau}(-\nu)^{-1} \alpha(\nu) \sigma(\nu: t) d \nu \\
& +\int_{-\infty}^{\infty} e^{-i \nu t} C_{\tau}(\nu)^{-1} \alpha(\nu) \sigma(-\nu: t) d \nu
\end{aligned}
$$

since $\alpha(-\nu)=\alpha(\nu)$ we see

$$
\Delta(t)^{1 / 2} f_{\alpha}\left(a_{t}\right)=2 \int_{-\infty}^{\infty} e^{-i \nu t} C_{\tau}(\nu)^{-1} \alpha(\nu) \sigma(-\nu: t) d \nu .
$$

Using classical results on the $\Gamma$-function we know that if $|\nu|>R, \operatorname{Im} \nu \leqq 0$, then

$$
\left|C_{\tau}(\nu)^{-1}\right| \leqq C(1+|\nu|)^{N}
$$

for some $C>0, N>0$. Integrating $e^{-i \nu t} C_{\tau}(\nu)^{-1} \alpha(\nu) \sigma(-\nu: t)$ about the contour $\Gamma_{R}$ :

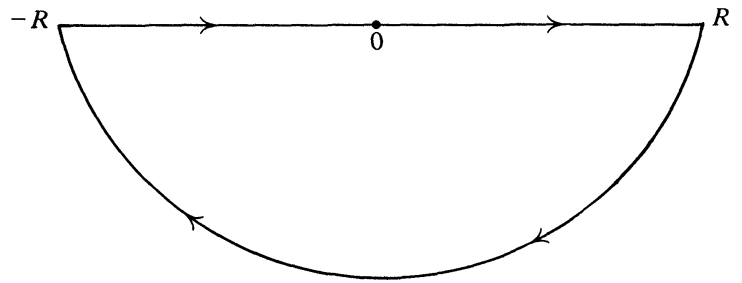

and using the above estimates on $C_{\tau}(\nu)^{-1}, \alpha(\nu), \sigma(-v: t), \operatorname{Im} \nu \leqq 0$ we find that if $t>T$,

$$
\Delta(t)^{1 / 2} f_{\alpha}\left(a_{t}\right)=4 \pi i \sum_{j=0}^{l_{\tau}} \operatorname{Re} s_{\nu=z_{1}} e^{-i \nu t} C_{\tau}(\nu)^{-1} \alpha(\nu) \sigma(-\nu: t) .
$$

Here $l_{\tau}$ is the greatest integer less than $(|k|-n) / 2\left(\tau=\tau_{k}\right)$ and

Notice that if $|k|<n$, then there are no $z_{j}$.

$$
z_{j}=i(2 j+n-|k|), \quad j=0, \cdots, l_{\tau} \text {. }
$$


To complete the proof we need

LEMMA 8.5. If $\nu=z_{j}, j=0,1, \cdots, l_{\tau}$, then

$$
\Delta(t)^{1 / 2} E_{\tau}\left(\nu: a_{t}\right)=e^{-i \nu t} C_{\tau}(-\nu) \sigma(-\nu: t) .
$$

This lemma is proved by a careful analysis of where the holomorphic discrete series appears in the $\pi_{\xi, \nu}, \nu \in C$. Using the fact that $f_{\alpha} \in L^{2}(G)$, we therefore see that $g \rightarrow E_{\tau}(\nu: g)$ is a matrix entry of an element of $\hat{G}_{d}$ for $\nu=z_{j}, j=0,1, \cdots, l_{\tau}$. Thus, if

$$
\phi_{j}(g)=4 \pi i \underset{\nu=z_{j}}{\operatorname{res}} C_{\tau}(\nu)^{-1} \alpha(\nu) E_{\tau}(-\nu: t),
$$

we see that $f_{\alpha}-\sum \phi_{j} \in C_{c}^{\infty}(\tau: G: \tau)$. This completes the proof of Theorem 8.1.

9. Applications to $\Gamma \backslash S U(2,1)$. We return to the notation of $\S 7$. We will study the case $G=S U(2,1)$ in this section.

Proposition 9.1. Let $\omega \in \hat{G}$ be such that if $\gamma \in \hat{K}$ and $\gamma$ is one dimensional, then $\left[\left.\omega\right|_{K}: \gamma\right]=0$. Let $\tau \in \hat{K}$ be such that $\left[\left.\omega\right|_{K}: \tau\right] \neq 0$. Set for $\theta \in \mathbf{R}$,

$$
m(\theta)=\left[\begin{array}{ccc}
e^{i \theta} & 0 & 0 \\
0 & e^{-2 i \theta} & 0 \\
0 & 0 & e^{i \theta}
\end{array}\right], \quad M=\{m(\theta) \mid \theta \in \mathbf{R}\} .
$$

Suppose that $\tau(m(\theta))$ diagonalizes with diagonal entries $e^{i k_{j} \theta}, j=1, \cdots, r$. Then there exists $f \in C_{c}^{\infty}(G)$ so that

(1) $\Theta_{\omega}(f)=1$;

(2) $F_{f}=0$;

(3) if $\eta \in \hat{G}$ has a different infinitesimal character than $\omega$, then $\Theta_{\eta}(f)=0$.

(4) if $\eta \in \hat{G}$ and $\left[\left.\eta\right|_{K}: \tau\right]=\left[\left.\eta\right|_{K}: \tau_{0}^{2 k_{j}}\right]=0, j=1, \cdots, r$, then $\Theta_{\eta}(f)=0$ (here we use the parametrization of representations of $U(2)$ given in \$7).

Proof. Let $\tau^{*}$ be the complex conjugate representation to $\tau$. Let $(\tau, V)$ be an irreducible unitary representation in the class of $\tau$. Choose

$$
h: G \rightarrow \operatorname{End}(V) \text { so that } h\left(k_{1} g k_{2}\right)=\tau^{*}\left(k_{1}\right) h(g) \tau^{*}\left(k_{2}\right)
$$

for $k_{1}, k_{2} \in K, g \in G, h$ of class $C_{c}^{\infty}$ and $v \in V$ so that if $\phi(g)=\langle h(g) v, v\rangle$ $(\langle$,$\rangle the K$-invariant inner product on $V)$, then $\Theta_{\omega}(\phi)=1$.

We compute $F_{\phi}(m(\theta) a)$.

$$
\begin{aligned}
F_{\phi}(m a) & =e^{\rho(\log a)} \int_{K \times N} \phi\left(k m a n k^{-1}\right) d k d n \\
& =e^{\rho(\log a)} \int_{N}\left(\left\langle\int_{K} h\left(k m a n k^{-1}\right) d k \cdot v, v\right\rangle\right) d n \\
& =e^{\rho(\log a)} \int_{K}\left(\left\langle\tau^{*}(k) h(\operatorname{man}) \tau^{*}(k)^{-1} d k \cdot v, v\right\rangle\right) d n .
\end{aligned}
$$

The Schur orthogonality relations for $K$ say that if $T \in \operatorname{End}(V)$, then

$$
\int_{K} \tau^{*}(k) T \tau^{*}(k)^{-1} d k=\frac{1}{d_{\tau}} \operatorname{tr} T,
$$


where $d_{\tau}$ is the dimension of $\tau$. Hence,

$$
F_{\phi}(m a)=\frac{1}{d_{\tau}} e^{\rho(\log a)}\langle v, v\rangle \cdot \operatorname{tr}\left(\int_{N} h(\operatorname{man}) d n\right) .
$$

Now if $m \in M$, then $n \rightarrow m n m^{-1}$ is a diffeomorphism of $N$ preserving $d n$. Hence,

$$
\tau^{*}(m) \int_{\mathrm{N}} h(a n) d n=\int_{\mathrm{N}} h(a n) d n \tau^{*}(m)
$$

for $m \in M$. Thus, if $V=\sum_{j=1}^{r} V_{j}$ with $\left.\tau(m(\theta))\right|_{v_{j}}=e^{i k_{j} \theta} I$, and if $P_{j}: V \rightarrow V_{j}$ is the orthogonal projection, then

$$
\int_{N} h(a n) d n=\sum_{j=1}^{r} P_{j} \int_{N} h(a n) d n P_{j}
$$

Set

$$
\psi_{j}(m(\theta) a)=\frac{1}{d_{\tau}} e^{\rho(\log a)}\langle v, v\rangle e^{-i k_{j}} \cdot \operatorname{tr} P_{j} \int_{N} h(a n) d n P_{j}
$$

Then $F_{\phi}(m a)=\sum_{j=1}^{r} \psi_{j}(m a), m \in M, a \in A$. Now

and

$$
F_{\phi}(m a)=F_{\phi}\left(m a^{-1}\right), \quad \psi_{j}(m(\theta) a)=e^{-i k_{j} \theta} \psi_{j}(a),
$$

$$
\psi_{j}(a)=\frac{1}{2 \pi} \int_{0}^{2 \pi} e^{i k_{i} \theta} F_{\phi}(m(\theta) a) d \theta .
$$

Hence, $\psi_{j}(a)=\psi_{j}\left(a^{-1}\right)$. Furthermore, it is easily seen that $\tau^{-2 k_{j}}(m(\theta))=e^{-i k_{i} \theta}$. Theorem 8.1 now implies that for each $j=1, \cdots, r$ there exists $\psi_{j} \in$ $C_{c}^{\infty}\left(\tau_{0}^{-2 k_{j}}: G: \tau_{0}^{-2 k_{j}}\right)$ so that $F_{\phi_{j}}=\psi_{j}$. Set $u=\phi-\sum_{j=1}^{r} \psi_{j}$. Then $u$ satisfies (1), (2), (4) of the proposition. From the results of $\S 7$ it is easily seen that the set $S=\left\{\eta \in \hat{G} \mid \Theta_{\eta}(u) \neq 0\right\}$ is finite. Let $\chi_{1}, \cdots, \chi_{m}$ be the distinct infinitesimal characters of the elements of $S$. Let $S_{i}=\{\eta \in S \mid \eta$ has infinitesimal character $\left.\chi_{i}\right\}$. We may (and do) assume that $\omega \in \chi_{1}$. Let 3 be the center of the complexified universal enveloping algebra, $U$, of $G$. Then if $\chi \rightarrow^{\prime} \chi$ is the antiautomorphism of $U$ defined by ${ }^{t} 1=1,{ }^{t} x=-x$ for $x \in(S)$, the Lie algebra of $G,{ }^{t}(x y)={ }^{t} y^{t} x$. Then if $z \in \mathcal{B},{ }^{t} z \in \mathcal{B}$, and if $\eta \in S_{i}, \Theta_{\eta}(z \cdot u)=\chi_{i}\left({ }^{t} z\right) \Theta_{\eta}(u)$. For each $i$, let $z_{i} \in 8, i=2, \cdots, m$, be such that $\chi_{1}\left(z_{i}\right) \neq \chi_{i}\left(z_{i}\right)$ (this is possible since $\chi_{1}, \cdots, \chi_{m}$ are distinct). Let

$$
z=\frac{\left(z_{2}-\chi_{2}\left(z_{2}\right)\right)\left(z_{3}-\chi_{3}\left(z_{3}\right)\right) \cdots\left(z_{m}-\chi_{m}\left(z_{m}\right)\right)}{\left(\chi_{1}\left(z_{2}\right)-\chi_{2}\left(z_{2}\right)\right) \cdots\left(\chi_{1}\left(z_{m}\right)-\chi_{m}\left(z_{m}\right)\right)}
$$

Then $\chi_{1}(z)=1, \chi_{i}(z)=0, i \geqq 2 . f={ }^{t} z \cdot u$ satisfies (1), (2), (3), and (4).

We now give some implications of Proposition 9.1. First of all let $\omega=T_{0}^{+}$. Then the $K$-types of $T_{0}^{+}$are $\tau_{p}^{3 p}, p \geqq 1$. Hence, $T_{0}^{+}$satisfies the hypothesis of Proposition 9.1. Take $\tau=\tau_{1}^{3}$. Then $\tau_{1}^{3}(m(\theta))$ diagonalizes with entries 1 and $e^{3 i \theta}$. Thus $k_{1}=0, k_{2}=3$. The representations with the same infinitesimal character as $T_{0}^{+}$are $1, T_{0}^{+}, T_{0}^{-}, D_{\mathrm{S}_{1} \mathrm{~S}_{2 \rho-\rho}}^{+}, D_{\mathrm{S}_{2} \mathrm{~S}_{1} \rho-\rho}^{-}, D_{-2 p}$. Let $f$ be as in 
Proposition 9.1. Now

$$
\begin{gathered}
{\left[\left.D_{-2 \rho}\right|_{K}: 1\right]=\left[\left.D_{-2 \rho}\right|_{K}: \tau_{0}^{6}\right]=\left[\left.D_{-2 \rho}\right|_{K}: \tau_{1}^{3}\right]=0,} \\
{\left[\left.D_{S_{2} s_{1} \rho-\rho}^{-}\right|_{K}: 1\right]=\left[\left.D_{S_{2} s_{1} \rho-\rho}^{-}\right|_{K}: \tau_{0}^{6}\right]=\left[\left.D_{S_{2} s_{1} \rho-\rho}^{-}\right|_{K}: \tau_{1}^{3}\right]=0,} \\
{\left[\left.T_{0}^{-}\right|_{K}: 1\right]=\left[\left.T_{0}^{-}\right|_{K}: \tau_{0}^{6}\right]=\left[\left.T_{0}^{-}\right|_{K}: \tau_{1}^{3}\right]=0 .}
\end{gathered}
$$

Hence, Proposition 9.1 combined with Theorem 6.7 implies that if $\Gamma \subset G$ is a discrete subgroup without elements of finite order other than $I$ so that $\Gamma \backslash G$ is compact, then

$$
N_{\Gamma}(1) \Theta_{1}(f)+N_{\Gamma}\left(T_{0}^{+}\right) \Theta_{T_{0}^{+}}(f)+N_{\Gamma}\left(D_{S_{1} s_{2} \rho-\rho}^{+}\right) \Theta_{S_{1} s_{2} \rho-\rho}(f)=f(I) \operatorname{vol}(\Gamma \backslash G) .
$$

Since $F_{f}=0, f(I)=\sum_{\omega \in \hat{G}_{d}} d(\omega) \Theta_{\omega}(f)$. Thus,

$$
f(I)=d\left(D_{S_{1} s_{2 \rho-\rho}}^{+}\right) \Theta_{D_{S_{1}}^{+} s_{2 \rho-\rho}}(f) \text {. }
$$

Now $\Theta_{T_{0}^{+}}(f)=1, \pi_{0}$ contains 1 and $T_{0}^{+}$and $\Theta_{\pi_{0}}(f)=0$ since $F_{f}=0$. Hence, $\Theta_{1}(f)=-1$. $\pi_{S_{1} S_{2} \rho-\rho}$ contains $T_{0}^{+}, D_{S_{1} S_{2}-\rho}^{+}$but not 1 . Hence, $\Theta_{D_{S_{1} s_{2} \rho-\rho}^{+}}(f)=-1$. Clearly $N_{\Gamma}(1)=1$. Hence, we have

$$
1-N_{\Gamma}\left(T_{0}^{+}\right)+N_{\Gamma}\left(D_{s_{1} s_{2} \rho-\rho}^{+}\right)=d\left(D_{S_{1} s_{2} \rho-\rho}^{+}\right) \operatorname{vol}(\Gamma \backslash G) .
$$

If we do the same argument starting with $\omega=D_{-2 \rho}$, then we find

$$
N_{\Gamma}\left(D_{-2 \rho}\right)-N_{\Gamma}\left(D_{S_{1} S_{2} \rho-\rho}^{+}\right)-N_{\Gamma}\left(D_{S_{2} S_{1} \rho-\rho}^{-}\right)-1=-d\left(D_{-2 \rho}\right) \operatorname{vol}(\Gamma \backslash G) \text {. }
$$

Here we note that if $\omega_{1}, \omega_{2} \in \hat{G}_{d}$ and $\omega_{1}, \omega_{2}$ have the same infinitesimal character, then $d\left(\omega_{1}\right)=d\left(\omega_{2}\right)$ (see Harish-Chandra [13]). Using Matsushima's formula $(\S 4,(\mathrm{ii}))$ and the list of $\S 7$, we find

LEMMA 9.2.

$$
\begin{array}{cc}
b_{0,1}(\Gamma \backslash G / K)=N_{\Gamma}\left(T_{0}^{+}\right), & b_{1,0}(\Gamma \backslash G / K)=N_{\Gamma}\left(T_{0}^{-}\right), \\
b_{0,2}(\Gamma \backslash G / K)=N_{\Gamma}\left(D_{s_{1} s_{2} \rho-\rho}^{+}\right), & b_{2,0}(\Gamma \backslash G / K)=N_{\Gamma}\left(D_{s_{2} s_{1 \rho-\rho}}^{-}\right), \\
b_{1,1}(\Gamma \backslash G / K)=1+N_{\Gamma}\left(D_{-2 \rho}\right) .
\end{array}
$$

Now the Gauss-Bonnet-Chern theorem (cf. Kobayashi-Nomizu [23, VII, §5]) implies that there is a normalization of $d g$ so that $\operatorname{vol}(\Gamma \backslash G)=\chi(\Gamma \backslash G / K)$ (the Euler number of $\Gamma \backslash G / K)$. This normalization is the Euler-Poincaré measure of Serre [337. This normalization of $d g$ is independent of $\Gamma$. Now

$$
\chi(\Gamma \backslash G / K)=1-b_{1}+b_{2}-b_{3}+b_{4} \quad\left(b_{i}=b_{i}(\Gamma \backslash G / K)\right)=2-2 b_{1}+b_{2}
$$

by Poincaré duality. Now $\Gamma \backslash G / K$ is Kähler. Hence, $b_{p, q}=b_{q, p}$. Hence, $b_{1}=2 b_{0,1}, b_{2}=b_{1,1}+2 b_{0,2}$. Thus,

$$
2-4 b_{0,1}+2 b_{0,2}+b_{1,1}=\operatorname{vol}(\Gamma \backslash G) \text {. }
$$

This says (in light of Lemma 9.2)

$$
3-4 N_{\Gamma}\left(T_{0}^{+}\right)+2 N_{\Gamma}\left(D_{S_{1} S_{2} \rho-\rho}^{+}\right)+N_{\Gamma}\left(D_{-2 \rho}\right)=\operatorname{vol}(\Gamma \backslash G) .
$$

Now using Lemma 9.2 on (1) and (2) and the fact that 
$d\left(D_{S_{1} S_{2} \rho-\rho}^{+}\right)=d\left(D_{-2 \rho}\right)$, we see

$$
\begin{gathered}
1-b_{0,1}+b_{0,2}=d\left(D_{-2 \rho}\right) \operatorname{vol}(\Gamma \backslash G), \\
b_{1,1}-1-b_{0,2}-b_{2,0}=-d\left(D_{-2 \rho}\right) \operatorname{vol}(\Gamma \backslash G) .
\end{gathered}
$$

Combining $\left(1^{\prime}\right),\left(2^{\prime}\right)$ and $(3)$, we find $d\left(D_{-2 \rho}\right)=\frac{1}{3}$.

Computing Chern classes from the $G$-invariant metric on $G / K$, we find that $C_{1}^{2}+C_{2}=4 E$ where $C_{1}, C_{2}$ are the first and second Chern classes and $E$ is the Euler class (actually this computation can be done on $P^{2}(C)$ ).

We have therefore proved

Theorem 9.3 (MAX Noether).

$$
1-b_{0,1}(\Gamma \backslash G / K)+b_{0,2}(\Gamma \backslash G / K)=\left(C_{1}^{2}+C_{2}\right)[\Gamma \backslash G / K] / 12 .
$$

Of course, this theorem is well known. If $\omega \in \hat{G}_{d}$ and if $\omega$ has infinitesimal character $\chi_{\Lambda}, \Lambda \in \mathscr{F}^{+}$(see Lemma 7.6), then $d(\omega)=C \Pi_{\alpha \in \Delta^{+}}(\langle\Lambda+\rho, \alpha\rangle /\langle\rho, \alpha\rangle)$ with $C$ depending only on $d g$ (see Harish-Chandra [13]). Since $d\left(D_{-2 \rho}\right)=\frac{1}{3}$ relative to the Euler-Poincaré normalization and $D_{-2 \rho}$ has infinitesimal character $\chi_{0}$, we see that $C=\frac{1}{3}$. We have also proved

LEMma 9.4. Let $d g$ be given the Euler-Poincaré normalization. If $\omega \in \hat{G}_{d}$ and $\omega$ has infinitesimal character $\chi_{\Lambda}, \Lambda \in \mathscr{F}^{+}$, then $d(\omega)=$ $\frac{1}{3} \Pi_{\alpha \in \Delta^{+}}(\langle\Lambda+\rho, \alpha\rangle /\langle\alpha, \alpha\rangle)$.

We now use the same technique on $T_{k}^{+}, k \geqq 1$, and find:

(5) If $k \geqq 1$, then

$$
N_{\Gamma}\left(D_{S_{1} S_{2}\left(k \Lambda_{1}+\rho\right)-\rho}^{+}\right)-N_{\Gamma}\left(T_{k}^{+}\right)=\frac{1}{6}(k+1)(k+2) \operatorname{vol}(\Gamma \backslash G) .
$$

Also if we start with the "lowest $K$-type" of $D_{S_{1} s_{2} s_{1}\left(k \Lambda_{1}+\rho\right)-\rho}$, we find

$$
\begin{aligned}
N_{\Gamma}\left(D_{S_{1} S_{2}\left(k \Lambda_{1}+\rho\right)-\rho}^{+}\right) & +N_{\Gamma}\left(D_{S_{2} S_{1}\left(k \Lambda_{1}+\rho\right)-\rho}^{-}\right) \\
& -N_{\Gamma}\left(D_{S_{1} S_{2} S_{1}\left(k \Lambda_{1}+\rho\right)-\rho}\right)=\frac{1}{6}(k+1)(k+2) \operatorname{vol}(\Gamma \backslash G) .
\end{aligned}
$$

Now if $k \geqq 2, D_{\mathrm{s}_{2} \mathrm{~S}_{1}\left(k \Lambda_{1}+\rho\right)-\rho}^{-}$is in $G_{d}^{\prime}$ (see Lemma 7.7). Hence, Theorem 3.2 implies:

(7) If $k \geqq 2$ then

$$
N_{\Gamma}\left(D_{S_{1} S_{2}\left(k \Lambda_{1}+\rho\right)-\rho}^{+}\right)=N_{\Gamma}\left(D_{S_{1} S_{2} s_{1}\left(k \Lambda_{1}+\rho\right)-\rho}\right) .
$$

We now give another application of this technique. Let $\omega=\pi_{-2 \Lambda_{2}}^{-}$. Then $\pi_{-2 \Lambda_{2}}=\pi_{-2 \Lambda_{2}}^{+} \oplus \pi_{-2 \Lambda_{2}}^{-}$. The elements of $\hat{G}$ with the same infinitesimal character as $\pi_{-2 \Lambda_{2}}^{-}$are $\pi_{-2 \Lambda_{2}}^{-}, \pi_{-2 \Lambda_{2}}^{+}, T_{-1}^{+}$. Arguing as above we find

LEMMA 9.5. $N_{\Gamma}\left(\pi_{-2 \Lambda_{2}}^{-}\right)-N_{\Gamma}\left(\pi_{-2 \Lambda_{2}}^{+}\right)=N_{\Gamma}\left(T_{-1}^{+}\right)$.

We note that (5) says for $k=1$,

$$
N_{\Gamma}\left(D_{S_{1} S_{2}\left(\Lambda_{1}+\rho\right)-\rho}^{+}\right)=N_{\Gamma}\left(T_{1}^{+}\right)+d\left(D_{S_{1} S_{2}\left(\Lambda_{1}+\rho\right)-\rho}^{+}\right) \operatorname{vol}(\Gamma \backslash G) .
$$


This is the reason why the series of representations $T_{k}^{ \pm}$was labeled with a $T$. It stands for "trash". The philosophy is that once the trash is "disposed of" (i.e., $\left.N_{\Gamma}(T)=0\right)$ then the formula for $N_{\Gamma}(\omega)$ should be $d(\omega) \operatorname{vol}(\Gamma \backslash G)$ for $\omega \in \hat{G}_{d}$. This "philosophy" is borne out by the following result.

THEOREM 9.6.

$$
\begin{aligned}
N_{\Gamma}\left(D_{S_{1} S_{2}\left(k_{1} \Lambda_{1}+k_{2} \Lambda_{2}+\rho\right)-\rho}^{+}\right) & =N_{\Gamma}\left(D_{S_{2} S_{1}\left(k_{1} \Lambda_{1}+k_{2} \Lambda_{2}+\rho\right)-\rho}^{-}\right) \\
& =N_{\Gamma}\left(D_{S_{1} S_{2} S_{1}\left(k_{1} \Lambda_{1}+k_{2} \Lambda_{2}+\rho\right)-\rho}\right) \\
& =\frac{1}{3} \prod_{\alpha>0} \frac{\left\langle k_{1} \Lambda_{1}+k_{2} \Lambda_{2}+\rho, \alpha\right\rangle}{\langle\rho, \alpha\rangle} \operatorname{vol}(\Gamma \backslash G)
\end{aligned}
$$

if $k_{1} \geqq 1$, and $k_{2} \geqq 1$.

The proof uses the same technique but starts with the "lowest $k$-type" of a nonunitary representation. Similar results were derived for more general groups in Hotta-Parthasarathy [17], using geometric techniques.

10. Asymptotic formulas. We conclude this article by discussing another technique for studying the $N_{\Gamma}(\omega)$. In this technique one studies the distribution of the $N_{\Gamma}(\omega)$ as $\omega$ varies over $\hat{G}$. Let $G$ be a connected semisimple Lie group with finite center. Let $\Gamma \subset G$ be a discrete subgroup so that $\Gamma \backslash G$ is compact. Let $K$ be a maximal compact subgroup of $G$. Let $Z(G)$ be the center of $G, Z(\Gamma)=Z(G) \cap \Gamma$. Let $\hat{K}_{\Gamma}$ be the set of all $\tau \in \hat{K}$ so that $\left.\tau\right|_{Z(\Gamma)}$ is the identity. For $\omega \in \hat{G}$, let $\lambda_{\omega} I$ be the value of the Casimir element on any representative of $\omega$.

Theorem 10.1 (Gelfand [8], Gangolli [6], Wallach [36]). There is a constant $C_{G}$ depending only on $G$ so that if $\tau \in \hat{K}_{\Gamma}$ and if $[Z(\Gamma)]$ is the number of elements in $Z(\Gamma)$ and $d=\operatorname{dim} G / K$, then

$$
\begin{aligned}
\sum_{\omega \in G} N_{\Gamma}(\omega)\left[\left.\omega\right|_{K}: \tau\right] e^{t \lambda_{\omega}}=C_{G} d_{\tau}[Z(\Gamma)](4 \pi t)^{-d / 2} \operatorname{vol}(\Gamma \backslash G)+o\left(t^{-d / 2}\right) \\
\text { as } t \rightarrow 0, t>0 .
\end{aligned}
$$

The value of the constant $C_{G}$ is just the volume of $K$ relative to the Haar measure that corresponds to the bi-invariant metric on $K$ gotten by restricting the negative of the Killing form of $G$ to the Lie algebra of $K$.

This formula was conjectured by Gelfand [8] and a proof of it for $G=S L(2, \mathbf{R})$ was sketched in Gelfand, Graev, Pyateckiï-Shapiro [9]. It was proved by Gangolli for the case $\tau=1$ and $G$ complex semisimple in [6]. Gangolli also conjectured the general form of this theorem in [6].

The idea of the proof is to observe that if $\Gamma$ has no noncentral elements of finite order, then $\Gamma \backslash G / K=X$ is a manifold. $\Gamma \backslash G \rightarrow \Gamma \backslash G / K$ is a principal bundle with structure group $K$. If $\tau \in \hat{K}_{\Gamma}$ then we can form the associated unitary vector bundle $\Gamma \backslash G \times{ }_{\tau} V_{\tau}=\mathbf{V}_{\tau}$. There is a natural connection on $\mathbf{V}_{\tau}$ and the connection Laplacian is just the Casimir operator, plus a scalar depending only on $\tau$. In this case the result is then a fairly easy generalization of the heat equation method (see McKean-Singer [28]) for studying the distribution of eigenvalues for a Laplacian. The general case is proved by 
developing an analogous theory for $G \backslash M$ where $M$ is a compact manifold and $G$ is a finite group acting on $M$. For details see Wallach [36].

This idea of using the heat equation method to study the $N_{\Gamma}(\omega)$ is due to Gangolli.

\section{REFERENCES}

1. J. Arthur, The trace formula for groups of Q-rank 1, Ann. of Math. (to appear).

2. A. Borel, Introduction to automorphic forms, Proc. Sympos. Pure Math., vol. 9, Amer. Math. Soc., Providence, R.I., 1966, pp. 199-210. MR 34 \#7465.

3. - Introduction aux groupes arithmétiques, Publ. Inst. Math. Univ. Strasbourg, XV, Actualités Sci. Indust., no. 1341, Hermann, Paris, 1969. Mr 39 \#5577.

4. M. Duflo and J-P. Labesse, Sur la formule des traces de Selberg, Ann. Sci. École Norm. Sup. (4) 4 (1971), 193-284.

5. N. Dunford and J. Schwartz, Linear operators. Vol. 3, Interscience, New York, 1972.

6. R. Gangolli, Asymptotic behaviour of spectra of compact quotients of certain symmetric spaces, Acta Math. 121 (1968), 151-192. MR 39 \#360.

7. - On the Plancherel formula and the Paley-Wiener theorem for spherical functions on semisimple Lie groups, Ann. of Math. (2) 93 (1971), 150-165. MR 44 \#6912.

8. I. M. Gel'fand, Automorphic functions and the theory of representations, Proc. Internat. Congr. Mathematicians (Stockholm, 1962), Inst. Mittag-Leffler, Djursholm, 1963, pp. 74-85. MR 31 \#273.

9. M. I. Gel'fand, M. I. Graev, and I. I. Pjateckiï-Šhapiro, Generalized functions. Vol. 6: Theory of representations and automorphic functions, "Nauka", Moscow, 1966; English transl., Saunders, Philadelphia, Pa., 1969. MR 36 \#3725; 38 \#2093.

10. Harish-Chandra, Representations of semi-simple Lie groups. VI. Integrable and squareintegrable representations, Amer. J. Math. 78 (1956), 564-628. MR 18, 490.

11. - Spherical functions on a semi-simple Lie group. I, II, Amer. J. Math. 80 (1958), 241-310, 553-613. MR 20 \#925; 21 \#92.

12. - Automorphic forms on semi-simple Lie groups, Lecture Notes in Math., no. 62, Springer-Verlag, New York and Berlin, 1968. MR 38 \#1216.

13. - Discrete series for semi-simple Lie Groups. II. Explicit determination of the characters, Acta Math. 116 (1966), 1-111. MR 36 \#2745.

14. - On the theory of the Eisenstein integral, Conf. on Harmonic Analysis (College Park, Md., 1971), Lecture Notes in Math., vol. 266, Springer-Verlag, Berlin and New York, 1972.

15. S. Helgason, Differential geometry and symmetric spaces, Pure and Appl. Math., vol. 12, Academic Press, New York and London, 1962. MR 26 \#2986.

16. - An analogue of the Paley-Wiener theorem for the Fourier transform on certain symmetric spaces, Math. Ann. 165 (1966), 297-308. MR 36 \#6545.

17. R. Hotta and R. Parthasarathy, Multiplicity formulae for discrete series, Invent. Math. 26 (1974), 133-178.

18. R. Hotta and N. R. Wallach, On Matsushima formula for the Betti numbers of a locally symmetric space, Osaka J. Math. (to appear).

19. K. Johnson, Paley-Wiener theorems for rank one groups (to appear).

20. H. Johnson and N. R. Wallach, Intertwining operators and composition series for the spherical principal series. I, Trans. Amer. Math. Soc. (to appear).

21. D. A. Kazdan, Connection of the dual space of a group and the structure of its closed subgroups, Funkcional. Anal. i Priložen. 1 (1967), no. 1, 71-74-Functional Anal. Appl. 1 (1967), 63-65. MR 35 \#288.

22. A. W. Knapp and E. M. Stein, Intertwining operators for semi-simple Lie groups, Ann. of Math. (2) 93 (1971), 489-578.

23. S. Kobayashi and K. Nomizu, Foundations of differential geometry. Vol. II, Interscience 
Tracts in Pure and Appl. Math., no. 15, Interscience, New York and London, 1969. MR 38 \#6501.

24. B. Kostant, On the existence and irreducibility of certain series of representations, Bull. Amer. Math. Soc. 75 (1969), 627-642. MR 39 \#7031.

25. R. P. Langlands, Dimension of spaces of automorphic forms, Proc. Sympos. Pure Math., vol. 9, Amer. Math. Soc., Providence, R. I., 1966, pp. 253-257. MR 35 \#3010.

26. Y. Matsushima, On the first Betti number of compact quotient spaces of higher-dimensional symmetric spaces, Ann. of Math. (2) 75 (1962), 312-330. MR 28 \#1629.

27. - A formula for the Betti numbers of compact locally symmetric Riemannian manifolds, J. Differential Geometry 1 (1967), 99-109. MR 36 \#5958.

28. H. P. McKean, Jr. and I. M. Singer, Curvature and the eigenvalues of the Laplacian, J. Differential Geometry, 1 (1967), no. 1, 43-69. MR 36 \#828.

29. G. D. Mostow, Strong rigidity for locally symmetric spaces, Ann. of Math. Studies, no. 78, Princeton Univ. Press, Princeton, N.J., 1973.

30. M. Raghunathan, Discrete subgroups of Lie groups, Ergebnisse der Mathematik und ihrer Grenzgebiete, vol. 68, Springer-Verlag, Berlin, 1972.

31. P. J. Sally, Jr., Analytic continuation of the irreducible unitary representations of the universal covering group of SL(2, R), Mem. Amer. Math. Soc. No. 69 (1967). MR 38 \#3380.

32. W. Schmid, On a conjecture of Langlands, Ann. of Math. (2) 93 (1971), 1-42.

33. J.-P. Serre, Cohomologie des groupes discrets, Prospects in Mathematics, Princeton Univ. Press, Princeton, N.J., 1971.

34. È. B. Vinberg, Some examples of crystallographic groups on Lobačevskiŭ spaces, Mat. Sb. 78 (120) (1969), 633-639= Math. USSR Sb 7 (1969), 617-622. MR 39 \#7497.

35. N. R. Wallach, Harmonic analysis on homogeneous spaces, Dekker, New York, 1973. 36. _- On an asymptotic formula of Gelfand and Gangolli for the spectrum of $\Gamma \backslash G, \mathrm{~J}$. Differential Geometry (to appear).

37. G. Warner, Harmonic analysis on semi-simple Lie groups. II, Die Grundlehren der math. Wissenschaften, vol. 189, Springer-Verlag, Berlin, 1972.

38. E. T. Whittaker and G. N. Watson, A course of modern analysis, 4th ed., Cambridge Univ. Press, Cambridge, 1927.

39. J. A. Morrow and K. Kodaira, Complex manifolds, Holt, Rinehart and Winston, New York, 1971.

Department of Mathematics, Rutgers University, New Brunswick, New Jersey 08903 